\title{
Neural Virtual Sensors for Adaptive Magnetic Localization of Autonomous Dataloggers
}

\author{
Dennis Groben, Kittikhun Thongpull, Abhaya Chandra Kammara, and Andreas König \\ Institute of Integrated Sensor Systems, EIT, TU Kaiserslautern, Erwin-Schrödinger-Straße 12, 67663 Kaiserslautern, Germany \\ Correspondence should be addressed to Andreas König; koenig@eit.uni-kl.de
}

Received 12 June 2014; Revised 16 October 2014; Accepted 19 October 2014; Published 30 December 2014

Academic Editor: Manwai Mak

Copyright (C) 2014 Dennis Groben et al. This is an open access article distributed under the Creative Commons Attribution License, which permits unrestricted use, distribution, and reproduction in any medium, provided the original work is properly cited.

\begin{abstract}
The surging advance in micro- and nanotechnologies allied with neural learning systems allows the realization of miniaturized yet extremely powerful multisensor systems and networks for wide application fields, for example, in measurement, instrumentation, automation, and smart environments. Time and location context is particularly relevant to sensor swarms applied for distributed measurement in industrial environment, such as, for example, fermentation tanks. Common RF solutions face limits here, which can be overcome by magnetic systems. Previously, we have developed the electronic system for an integrated data logger swarm with magnetic localization and sensor node timebase synchronization. The focus of this work is on an approach to improving both localization accuracy and flexibility by the application of artificial neural networks applied as virtual sensors and classifiers in a hybrid dedicated learning system. Including also data from an industrial brewery environment, the best investigated neural virtual sensor approach has achieved an advance in localization accuracy of a factor of 4 compared to state-of-the-art numerical methods and, thus, results in the order of less than $5 \mathrm{~cm}$ meeting industrial expectations on a feasible solution for the presented integrated localization system solution.
\end{abstract}

\section{Introduction}

Sensor networks have established themselves in scientific, industrial, and military applications. Military reconnaissance and surveillance, for example, employing distributed microphone or hydrophone arrays, can be named here. In the last decades, numerous nonmilitary, commercial applications could be observed, which incubated the creation of more and more powerful and increasingly smart and networked data loggers. The development was boosted by the soaring progress in microelectronics and MEMS technology, sensor technology, communications, and computational intelligence with a strong emphasis on artifical neural systems. This advanced the integration of wireless, autonomous, or even self-sufficient multisensor systems and sensor networks and opened, due to miniaturization and cost-effectiveness, new application fields from process control and automation to Ambient-Intelligence, for example, [1], Internet-of-Things (IoT), Cyber-Physical-Systems (CPS), Cyber-Physical-Production Systems (CPPS), Industrie4.0, and numerous biomedical tasks. The key vision for this development is the concept of Smart-Dust, introduced, for example, by the University of Berkeley $[2,3]$, which formulates the idea to integrate in a cubic millimeter multisensors, processing, communication, energy supply, and potentially actuators to a Smart-Sensor corresponding networks of numerous such units. The ITRS roadmap [4] clearly points out the advantageous development of microelectronics, MEMS, sensors, and packaging technology, for example, 3D integration technology, in particular on the so-called More-than-Moore direction of development [5] for Systems-in-Package ( $\mathrm{SiP}$ ) solutions, which allows the increase of information processing in the named nodes themselves. More and more complex methods of computational intelligence, neural networks, and learning systems, thus, become amenable for the realization of distributed intelligent integrated sensory systems or sensory swarms. Though the Smart-Dust vision has not completely become reality, the named advance in micro- and nanotechnologies already allows with regard to sensory diversity/bandwidth, achievable measurement uncertainty, and power dissipation minimization the realization of suitable sensor swarms for 
distributed measurement in industrial applications in the measurement and instrumentation field $[6,7]$.

One important aspect in the practical application of sensory networks or swarms with multisensory process parameter registration, for example, of temperature, pressure, humidity, conductivity, or impedance spectrum, is the logging of time and location context. A synchronized timebase, potentially addional location sensors, and apt signal processing are required for the task of context acquisition. Evaluation of distributed swarm measurements will be of value only, if time and location of the registration are available for swarm data visualization and analysis. In many application scenarios, including indoor, outdoor, or even maritine tasks, the radio signal itself and its attenuation (Received-SignalStrength-Indicator (RSSI)) can serve as sensory source and baseline for localization.

However, in industrially relevant scenarios of closed, large-scale containers, for example, stainless-steel fermentation tanks in brewery industry shown in Figure 1, strong attenuation in the medium and reflections from container walls hamper communication itself, and, thus, also localization.

In our research, an alternative localization system has been conceived to locate the units of the sensor swarm based on magnetic technology and inspired by existing tracking systems and technology, for example, helmet tracking in military applications or instrument or pill tracking in medical applications. The developed approach and system scales to the required industrial tank sizes. It employs integrated magnetic sensors together with algorithms from computational intelligence to robustly calculate the sensor node localization from magnetic field emitting beacons. In this paper, the basic electronic system and an extension by neural networks as virtual sensors in a supervised approach for the significant improvement of the localization quality and the simplified adaptation to changed or new application scenarios are presented. In Section 2, we will outline our approach, system architecture and electronics, scenarios, data acquisition, and the synchronization approach with the corresponding classification experiments. In Section 3 virtual sensor methods and corresponding parameter settings will be given. In Section 4, before concluding, our experiments and results will be presented.

\section{Magnetic Localization System}

Magnetic localization systems are in widespread use since more than two decades and related publication and patent activity, for example, $[8,9]$, can be observed. Numerous concepts and systems solutions can be found, which achieve a localization or a tracking of one or potentially several objects. These systems can be conceived in two dual ways by either employing an potentially integrated, magnetic marker, which is located by an external stationary sensor system or sensor array, or alternatively employing a plurality of external stationary magnetic field generator units, working as beacons, which are detected by magnetic sensors within the regarded mobile sensory nodes. The first approach is commonly employed in medical tracking systems, for

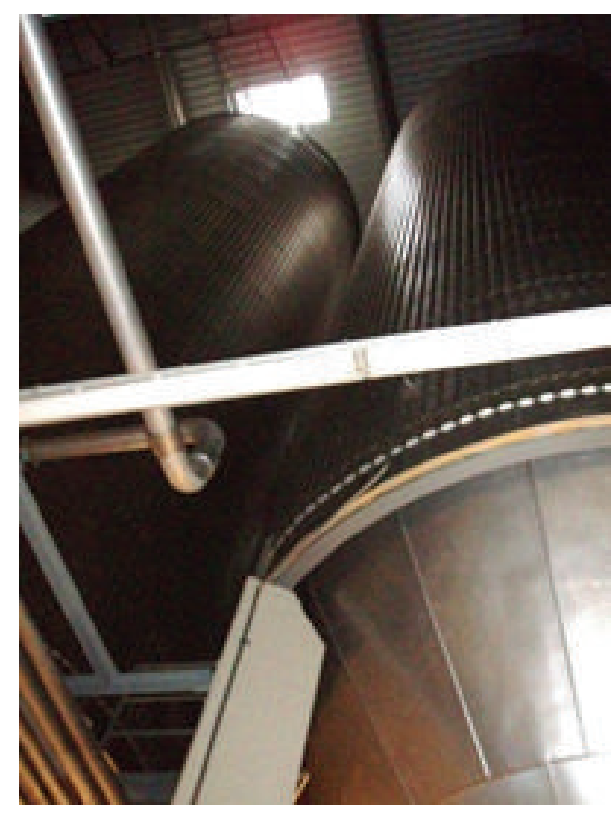

FIgURE 1: Fermentation tanks example of twenty meters height and six meters diameter from brewery industry (Courtesy Warsteiner).

example, in systems of Motilis [10] or Matesy [11], which employ permanent magnets as markers, for example, in a pill size arrangement moving through the human body. Tracking takes place at rather short distance. Application to a sensor swarm faces difficulties, as the discerning of multiple markers, that is, sensor nodes in the swarm, has not been satisfactorily solved so far. Naturally, instead of deploying permanent magnets in the sensory nodes, also actively controlled magnetic field generation by coil setups or spinning magnet setups could have been considered. However, with regard to size and power budget of autonomous miniaturized systems. The second approach with active beacons and integrated magnetic sensing in the swarm nodes, thus, is preferred in most applications, for example, in systems Polhemus [12] and Ascension [13] as well as medical applications of instrument or endoscopic task tracking. Also, this variant is more fortunate for and compatible with the development of Smart-Pills, for example, in the Vector-project [14]. Basically, two beacon control methods can be distinguished, employing AC-fields (e.g., systems of Polhemus [12]) or DC fields (e.g., systems of Ascension [13]), which in the latter case means that the beacons work in a time multiplexing scheme; that is, each beacon emits a field at a defined time frame, which allows the sensor and the the localization algorithms to uniquely identify the source. However, this feature also gives rise to the synchrony of the beacon control and sensor swarm units time bases, that is, requiring an approach for maintaining synchronization.

AC-field generation additionally, allows the use of frequency multiplexing, that is, the simultaneous activity of several beacons at different frequencies. However, this requires more effort on the receiver side. Also, quasi-DC approaches are less vulnerable to the presence of magnetic materials and eddy-current induced fields. 


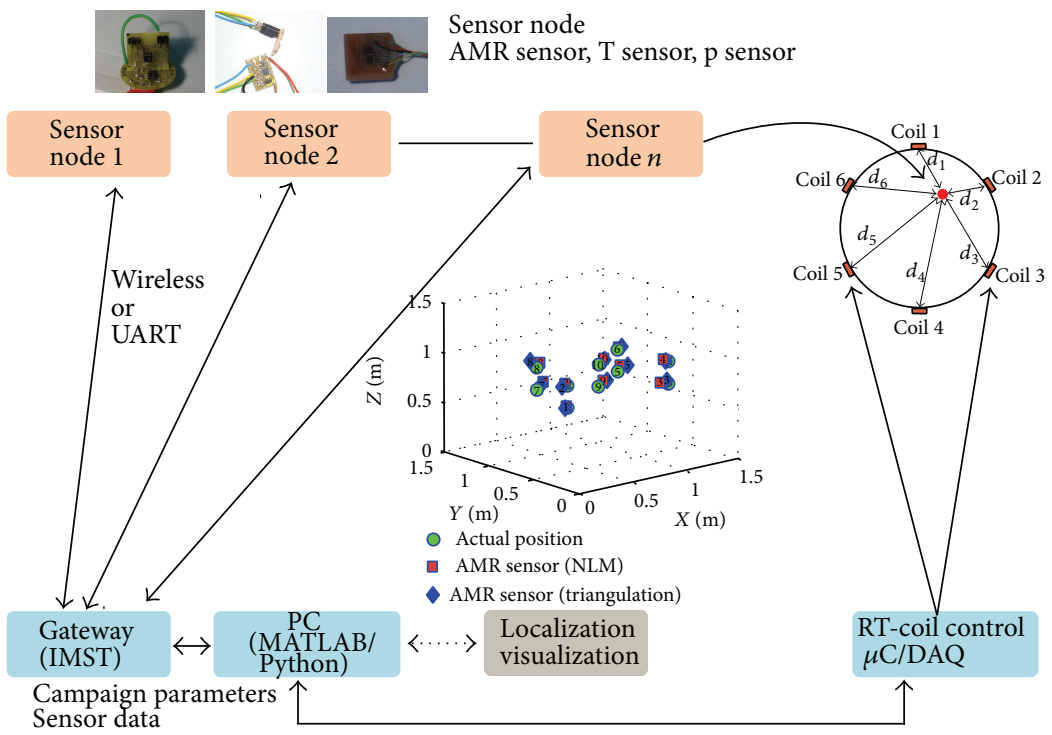

FIGURE 2: The block diagram shows the implemented system and physical structure of the experimental setups.

The various system solutions, for example, for indoor localization [15-18] or underground animal tracking [19] and so forth, have employed magnetic sensors from the spectrum of available principles, for example, SQUIDs, miniaturized coils, Hall elements, for example, [20], field plates, or magnetoresistive sensors with various operating principles, summarized as XMR sensors. Compass sensors, for example, for the mobile phone market, or automotive tasks have incubated the conception of new sensory units, for example, with 9 axes, including acceleration, Gyros, and magnetic sensors in 3D arrangement [21, 22]. These rather novel chips on the market will support the compact implementations of the system and approach presented in this paper even better. For reasons of sensitivity, that is, due to weak beacon fields or large distances between sensor and beacon, AnisotropeMagneto-Resistive (AMR) sensors are very attractive as they feature high sensitivity and offer favorite linearity properties and temperature range, as well as small size and low-power operation.

2.1. Pursued Magnetic Localization Approach. In our research, based on the outlined state-of-the-art and the particular constraints of the regarded application domain, a magnetic localization system, including synchronization capability, for a sensor swarm has been developed. The system employs the concept of quasi-DC field generation by stationary field generating beacons, realized by dedicated coils and suitable computer controlled power electronics. Further, AMR sensors AFF755B [23] of Sensitec have been employed in 3 axial 3D arrangement, which has been integrated by various packaging technologies [24], for example, standard PCB, or Active-Multi-Layer (AML) [25].

Figure 2 shows the block diagram of our magnetic localization system, which has been implemented and employed in four different demonstrators.
In our 3D-AMR magnetic sensor, each AMR channel is connected to an AD 8290 instrumentation amplifier, which provides a gain $G$ of 50 , control of the bridge, and power-down of bridge and amplifier under control of the microcontroller (atmel XMEGA256A3). Three such channels serve for $X, Y$, and $Z$ registration and sampling takes place in a ternary scheme of the exciting magnetic field, computing the mean $V_{m}$ of positive and negative phase voltages, $V_{i}^{p}$ and $V_{i}^{n}$, in (1). Only the magnitude of the flux density $B_{m}$ is required (see (2) and (3)):

$$
\begin{gathered}
V_{i}=\frac{V_{i}^{p}-V_{i}^{n}}{2}, \quad i=\{x, y, z\}, \\
V_{m}=\sqrt{V_{x}^{2}+V_{y}^{2}+V_{z}^{2}}, \\
B_{m}=\sqrt{B_{x}^{2}+B_{y}^{2}+B_{z}^{2}}=\frac{V_{m}}{S \cdot V_{s} \cdot G} .
\end{gathered}
$$

Here, $G=50$, the bridge supply is $V_{S}=3.3 \mathrm{~V}$, and the sensitivity value $S$ is taken from the Sensitec datasheet with the typical value of 11.7, which advocates the calibration of $S$ for each sensor instance. In our work, we will comprise this step by neural network learning. In (4), Bio-SavartsLaw is employed to establish a relationship between the flux density values $B_{m}$ obtained from measurement and the aspired distances $d$ by resolving for the distance $d$ in (5):

$$
\begin{gathered}
B_{m}=\frac{\mu_{0}}{2} \cdot \frac{n \cdot I \cdot R^{2}}{\left(R^{2}+d^{2}\right)^{3 / 2}}, \\
d=\sqrt{\left(\frac{(1 / 2) \cdot \mu_{0} \cdot n \cdot R^{2} \cdot I}{B_{M}}\right)^{2 / 3}-R^{2} .}
\end{gathered}
$$

Here, $\mu_{0}$ is the permeability in vacuum, $n$ is the number of windings of the coils, $I$ is the DC current driving the coil, 
TABLE 1: Technical details of conceived demonstrators.

\begin{tabular}{lccc}
\hline & $\begin{array}{c}\text { ISE } \\
\text { demonstrator }\end{array}$ & $\begin{array}{c}\text { Brewery } \\
\text { demonstrator }\end{array}$ & $\begin{array}{c}\text { HMI } \\
\text { demonstrator }\end{array}$ \\
\hline Dimensions [cm] & D210 $\times$ H180 & D220 $\times$ H300 & D $12 \times$ H60 \\
Number of coils & 6 & 12 & 6 \\
Coil diameter [cm] & 12.5 & 32 & 12 \\
Number of windings & 100 & 180 & 120 \\
Coil current in [A] & 5 & 3 & 3 \\
Coil placement & Cylindrical & Cylindrical & Spherical \\
Number of coil rings & 2 & 3 & 2 \\
\hline
\end{tabular}

and $R$ is the coil radius. As this simple model only works fine, if the sensor is located on the principle coil axis and the sensor instance sensitivity is known, the estimated distances can be out of scale, which is fixed in a first processing step by a heuristic global correction or scaling factor $\alpha$ added in (6). The computation of $\alpha$ is explained in Section 2.3.2(e):

$$
d=\alpha \cdot \sqrt{\left(\frac{(1 / 2) \cdot \mu_{0} \cdot n \cdot R^{2} \cdot I}{B_{M}}\right)^{2 / 3}-R^{2}} .
$$

Thus, RSSI equivalent distance values are obtained for the case of the magnetic localization system and can be treated with the standard methods given in Section 2.3.

2.2. Demonstrator and Integration Issues. In order to validate and optimize the localization system under changing environmental condition and different scale, three experimental setups or demonstrators were conceived.

The first demonstrator is located in our ISE (integrated sensory systems) lab. Six coils are placed in a cylindrical fashion similar to the shape of a steel tank. The six coils are separated into two rings of three coils each on two levels, rotated by an angle of $60^{\circ}$. An $x$-y-movable sledge with attached scales serves for ground truth position acquisition. Relevant technical data is surveyed in Table 1. In the following, this setup is referred to as ISE demonstrator.

The brewery demonstrator (see Figure 3 ) is a real brewery container in smaller scale. It was temporary set up at Technikum Warstein to validate the localization system in the industrial environment where parasitic magnetic fields of, for example, large pumps or other heavy machinery might cause problems. The dimensions are bigger than those of the ISE demonstrator, so in total 12 coils separated into 3 rings of 4 coils each were installed. The coils itself were also larger in diameter. The coil positions were appropriately determined as a mandatory baseline for localization. Relevant technical details are summarized in Table 1. A suiting reference system for ground truth sensor position determination was established inside the tank, which for obvious reasons was not filled with liquid in these experiments. Also, acquiring a larger number of points than the registered 30 locations without proper climbing support was not easy, especially within the limited duration of availability of the experimental setup for this work.

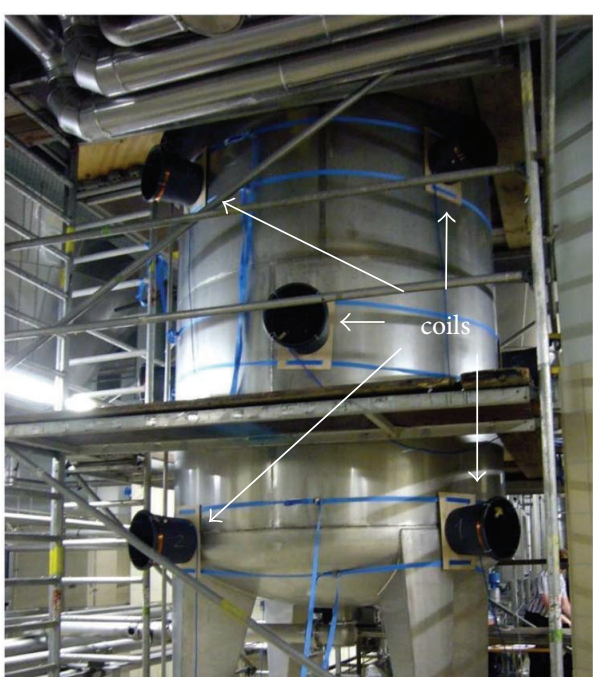

FIGURE 3: The coils of the brewery demonstrator are separated into three rings of four coils each.

Here, a lower limit for the achievable localization error is in particular given by the uncertainty of the actual sensor center determination.

Additionally, the obtained data matches well with data acquired from the target industrial process. The reason for this is that the experimental tank was placed in close proximity to the production environment including fermentation tanks, so it can be rightfully assumed that it was subject to the same sources of influence like pumps, heaters, coolers, and so forth. Due to the random nature of the switching activity of these devices with regard to the coil switching of our localization scheme, as well as enforced confidentiality on process details, a comprehensive investigation is very difficult. But, due to our ternary switching of the coil fields and ensuing differential processing in the sensory nodes, all sources of influence quasiconstant in each coil's time slot will be significantly suppressed or even canceled out.

The last demonstrator is a small, down-scaled, and mobile version of our localization system and was specially designed and build for the presentation at Hannover Messe Industrie (HMI) 2013. Figure 4 shows the demonstrator at our booth with the electronics on the table and a monitor showing a MATLAB GUI in the background. The tank is modeled by a tube of acrylic glass. Also the holding construction of the six coils is made of this material. See Table 1 for comparison to the other demonstrators. This mobile demonstrator will be called HMI demonstrator in the following.

The coil placement, similar to the general problem of anchor node placement in wireless sensor networks [26, 27], was an important consideration in our work. While a symmetrical distribution of the coils is not essential, equidistant placement of the coils helps in making sure that in every possible sensor position in the tank there is a minimum of three coils that generate fields of sufficient strength for proper localization. Also significant asymmetrical positioning of the coils will aggravate the angle problem discussed in Section 2.1 and will proportionally degrade the localization accuracy. We 


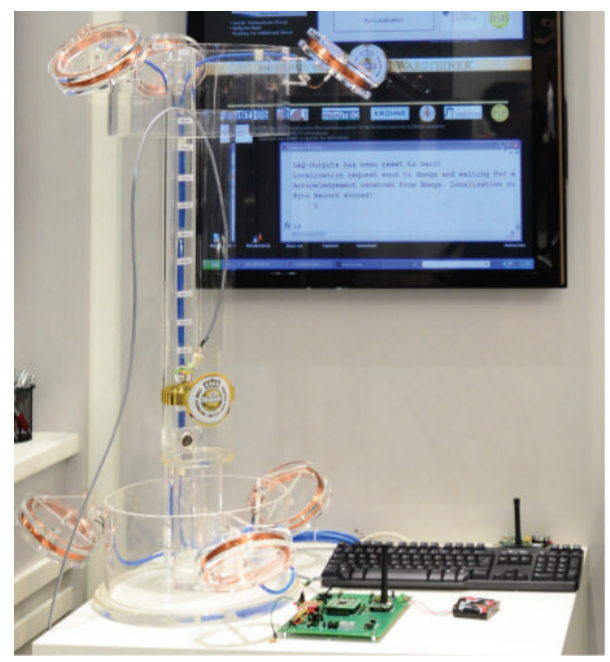

FIgURE 4: The HMI demonstrator features spherically placed coils to minimize the off-axis error of the coils. The actual AMR-sensor module in the current integration state of the prototype is wired to the sensor development board located outside the acrylic glass tube.

investigated 3 different topologies parallel planes, cylindrical, and ellipsoidal placement of coils. The HMI demonstrator has a very unfortunate aspect ratio and scale, which could be partly compensated by the most promising ellipsoidal coil arrangement.

The overall goal of the described research is the achievement of a compact 3D integrated data logger for a sensor swarm in distributed measurements. In the first development step, standard printed-circuit board (PCB) version of 3DAMR-sensor (see Figure 5 (left)) [28] and the complete datalogger (see, e.g., Figure 4) have been conceived. The 3DAMR-sensor has already seen implementation in various 3D integration technologies [24]. Figure 5 (right) shows the example of the Active-Multilayer-Technology (AML) first version with a first-cut design size of $16 \mathrm{~mm} \times 17 \mathrm{~mm} \times 5 \mathrm{~mm}$. The bulky connectors visible in Figure 5 are required only for the modular development systems and, of course, will be obsolete for the integrated target system. Further substantial size reduction by 3D layout optimization can be expected. AML technology is one favorable option to encapsulate and integrate the complete aspired data logger.

\subsection{Standard Localization Algorithms}

2.3.1. Standard Algorithms in Wireless Sensor Systems. In the majority of wireless sensor systems, RF-communication signals serve as the information source for the localization approaches. The RSSI, again, is the most common indicator to estimate the distance between a sender and receiver pair in the network, for example, a stationary beacon and a sensor node. Based on four or more distance estimates, quite similar to data visualization approaches, triangulation, multilateration [29-31], or multidimensional scaling (MDS) methods, in particular the nonlinear Sammon's mapping (NLM), [32] are employed in standard approaches for sensor location

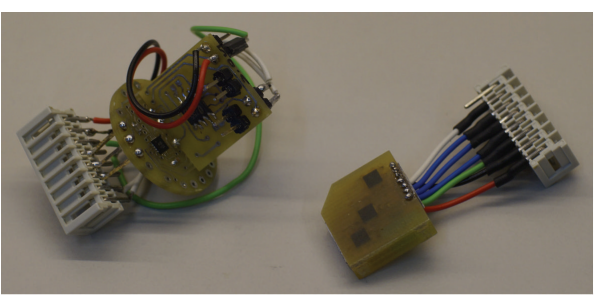

FIGURE 5: Regarded 3D-AMR-sensor implementations: standard PCB with AFF755B (left) and AML technology node with AFF756 (right).

or coordinates estimation. Sammon's iterative mapping is computationally demanding and requires a postprocessing step, denoted as conformal mapping, to compute the actual absolute coordinates from the relative information. Further, employed gradient descent technique might not converge to the best solution. The computationally fortunate multilateration, which is based on least squares optimization or MoorePenrose pseudoinverse, or the standard NLM both work fine for the magnetic system pursued here and the localization results of these unsupervised state-of-the art methods will be compared to those of the newly proposed ones.

2.3.2. Enhanced Algorithms for Wireless Sensor Systems. The issues of high computational complexity $O\left(N^{2}\right)$, potential local optimum solution, and required post processing for absolute coordinate obtainment motivated the recent development of an advanced approach. In the particular scenario faced here, mobile sensors individually have to estimate their position with regard to stationary beacons of known number and position. This can be tackled well with the NLM recall (NLMR) variant [33], reducing the computational complexity to $O(N)$ and immediately returns absolute coordinates. This approach along with advanced optimization methods for achievement of better solution quality has been introduced in [34] and will also serve, in the variations briefly outlined below for a self-contained presentation, for comparison and extension of the newly proposed localization methods.

(a) NLMR. The NLMR for localization as introduced in [34] has the following simplified cost function $E_{i}(m)$ with $m$ as step or iteration variable:

$$
E_{i}(m)=\frac{1}{c} \sum_{j=1}^{K} \frac{\left(d_{X i j}-d_{Y i j}(m)\right)^{2}}{d_{X i j}},
$$

where

$$
d_{X i j}=\sqrt{\sum_{q=1}^{m}\left(v_{i q}^{r}-v_{j q}^{t}\right)^{2}}, \quad c=\sum_{j=1}^{K} d_{X i j},
$$

where $d_{X i j}$ is the distance between the currently mapped recall datum and the $K$ previously mapped training data samples in the high dimensional space. The distances in the new space can be found using standard or advanced optimization methods. 
(b) Gradient Descent. The gradient descent technique for NLMR is from $[33,34]$. The equations are

$$
y_{i q}(m+1)=y_{i q}(m)-\operatorname{MF} \times \Delta y_{i q}(m)
$$

with

$$
\Delta y_{i q}(m)=\frac{\left(\partial E_{i}(m) / \partial y_{i q}(m)\right)}{\left(\partial E_{i}^{2}(m) / \partial y_{i q}(m)^{2}\right)}, \quad 0<M F \leq 1
$$

where $y_{i q}(m+1)$ is the new position, MF is the magic factor which reduces with time, $y_{i q}(m)$ is the current position and $E_{i}(m)$ is the cost function at the current position. MF is initialized to 1 . We keep reducing the MF by $10 \%$ everytime we find a better fitness.

(c) NLMR-Simulated Annealing. We use the basic simulated annealing [34] where we start with a relatively high temperature $\left(T_{0}=1\right)$ which is reduced $\left(T x=T_{(x-1)} * 0.8\right)$ over the number of cycles and reduce the chances of choosing a bad solution as the temperature decreases (accept any solution if $\left.p(0,1)<t_{x}\right)$. The new solutions are found by a Markov chain shown in (9) with a random MF between $-e_{0}$ and $e_{0}$ where $e$ (energy factor) reduces over time. The algorithm runs for 1000 iterations to get the best solutions. The number of iterations required was found heuristically.

(d) NLMR: Particle Swarm Optimization. Standard particle swarm optimization described in [35] is used with $C_{1}=$ $C_{2}=2$ and without inertia. Having no inertia helped in faster convergence of the algorithm. 300 particles were used with 150 generations to find the best results in the experiments.

(e) Correction Factor $\alpha$. A scale error in distance estimation became obvious from measurements that is introduced by the model (see (3) to (5)). This reduces the accuracy of the algorithms. Multilateration is mostly robust to this scale error because it uses only the differences in distance and not absolute distance, while the other methods require a correction. The correction factor $\alpha$, shown in (6), can be found by determining the ratio of the model estimated and actual distances. We investigated suitable $\alpha$-settings by computing this ratio for all samples of each data set and found that there was only $\approx 1 \%$ variation in the result. So, in the simplest case just taking one representative sample to compute the correction factor already significantly improves the results. More sophisticated search strategies to find the correction factor, for example, using unsupervised hyperheuristics, will be considered for future work.

2.4. Data Acquisition. The data sets acquired from the three described demonstrators for the ensuing experiments have been collected by either a wired standard or a wireless proprietary measurement system. The wired one is a Data Translation DT9816 data acquisition board (DAQ), which is controlled by MATLAB. The 3D-AMR-sensor module is directly wired to the DAQ, and the amplified sensor voltages are measured by analog input ports with 16-bit ADCs and are immediately available in MATLAB for signal processing
TABLE 2: Representative data sets acquired from the demonstrators of Table 1 for the experiments.

\begin{tabular}{lccc}
\hline & $\begin{array}{c}\text { SEL }_{1,2} \\
\text { data set }\end{array}$ & $\begin{array}{c}\text { BREW } \\
\text { data set }\end{array}$ & $\begin{array}{c}\text { HMI data } \\
\text { set }\end{array}$ \\
\hline $\begin{array}{l}\text { Demonstrator } \\
\text { Sensor node }\end{array}$ & ISE & Brewery & HMI \\
Sensor type & AFF755B & Std. PCB & AMF755B \\
DAQ system & DT9816 & DT9816 & $\begin{array}{c}\text { XMEGA } \\
\text { 256A3 }\end{array}$ \\
ADC resolution & 16 bit & 16 bit & 12 bit \\
Coil control & DT9816 & XMEGA & XMEGA \\
Number of & & 256 A3 & 256 A3 \\
samples/plateau & 10000 & 10000 & 128 \\
Number of positions & 169 & 30 & 44 \\
Number of repetitions & 0 & min. 10 & 3 \\
Total number of trials & 169 & 325 & 132 \\
\hline
\end{tabular}

and localization computation. The wireless system (see also Figure 2) corresponds to the target architecture of the final data logger, which in the first step has been implemented as a modular development PCB system. This development board (see Figure 4) features process sensors and a radio module for host PC via a gateway communication, for example, for configuration and measured process data transfer. For conversion of the 3D-AMR-sensor voltages the 12-bit ADC of the $\mu \mathrm{C}$ atmel XMEGA 256A3 is used in time-multiplex. Table 2 gives an overview of the three representative data sets chosen as the baseline for the following investigations. The ISEL data, in particular, serves with a $13 \times 13$ equidistant spatial sampling with a a $10 \mathrm{~cm}$ pitch in a plane for the elucidation of the spatial localization error distribution. ISEL was recorded two times with two different sensor instances, which will be denoted as ISEL $_{1}$ and ISEL $\mathrm{In}_{2}$ in the following.

Figure 7 shows raw data from the ISEL data sets. The noise level is quite substantial in comparison to the actual signal. High frequent noise is alleviated by multiple sampling of each DC plateau, for example, 10,000 times, and computing the plateau mean. This approach has been chosen instead of a low pass filter because the edges of the magnetic DC plateaus also serve as synchronization signals and thus have to be as steep as possible. Other sources of error, for example, stationary magnetic fields, as the earth magnetic field, can be canceled out by either standard AMR-sensor flipping or the ternary coil switching introduced in this work, which is more efficient from the point of view of energy conservation in the sensory node [28].

The substantially lower resolution of the $\mu \mathrm{C}$ ADC required a more sophisticated read-out approach to avoiding loss of distance resolution. A zooming technique was applied that by differential measurement, offset autozeroing, and scaling to full scale makes maximum use of the $\mu \mathrm{C} \mathrm{ADC}$ 12-bit resolution [24]. Thus, competitive localization results to the DAQ could be achieved on the integrated data logger target platform, which was employed to acquire HMI data set. 
2.5. Synchronization Issues. The introduced magnetic localization concept and system crucially depends on the knowledge of the timing of each coil's activation in the respective autonomous wireless sensor node. This requires a synchronization between the clock in the coil switching unit and the clock in each sensor node. As timebases commonly show a significant uncertainty, in particular, when they are expected to be small, cheap, and low-power, repeated synchronization is required. In our case, the tolerable or recoverable deviation limit is determined by $50 \%$ of the duration of a single coil's switching cycle. In wired versions, the synchronization information easily can be made available by an extra trigger line. Also, in RF-based wireless sensor networks synchronization can be achieved by communications. However, in the given container scenario, deficiencies of RF hamper data communication in general and localization as well as synchronization in particular. A very straightforward idea is now to derive the coveted synchronization information also from the emitted magnetic field. Indeed, this has been invented already in [8], however, with the sensor, denoted as sync pick-up coil, stationarily located very close to the emitting coil and attached by long wires to the sensor itself. A lock-in amplifier is used for the synchronization step there.

The industrial scenario investigated in our case does not allow such a fortunate arrangement. The magnetic sensor employed for localization has to be employed also to measure the data for synchronization and, thus, is remote and at varying distances and orientations with regard to each of the coils. This scenario aggravation requires additional engineering effort. A resource hungry sampling of a sufficient time window around the expected first rising edge in the magnetic field has to be carried out. In the very first-cut solution, a heuristic threshold detector had been conceived in our previous work, which detects the magnetic field rising edge of a coil being switched on. Based on the difference of the detected edge and timestamp to the expected timestamp the local wireless autonomous sensor node's clock will be cyclically readjusted. The underlying technical problem of finding an edge or pulse in substantially noisy electrical or magnetic field data has been visited before in communications, networking, and, most important, magnetic head data reading in mass storage, for example, in [36]. The task mentioned last comes closest to our interest and activities. In the work presented here, we investigate the SVM classifier (SVM-C) technique as trainable edge detector. It is applied with the parameter settings of unnormalised data obtained from the learning task of $C=10,000$ and kernel function is RBF with $\gamma=0.01$. The input feature space is represented by a 2,000 samples wide sliding window at an increment of 250 samples and the output of the classifier represents the two classes "edge" and "no edge." The processing structure of SVM based edge detection system is illustrated in Figure 9. The experimental data for the synchronization investigations has been extracted from the wireless sensor node prototype in the HMI demonstrator in continuous sampling mode; that is, whole localization cycles were sampled, whereas in contrast to this, for localization only parts of the coil switched-on plateaus have to be sampled. Three recorded raw data sets ("syncrawl," "syncraw2,"
TABLE 3: Edge classification results.

\begin{tabular}{lcc}
\hline Method & Heuristic & SVM-C \\
\hline Generated edges & & 66 \\
Detected edges & $35(53.03 \%)$ & $41(62.13 \%)$ \\
Missed edges & $31(46.97 \%)$ & $25(37.69 \%)$ \\
Spurious detections & $14(21.21 \%)$ & $0(0.0 \%)$ \\
\hline
\end{tabular}

and "syncraw3") of localization cycle, each contains 262,144 samples of acquired ADC 12-bit values, were used in the experiment. Each raw data set contains 33 different "edge" shapes and levels and 1,028 "no edge" events, including some spurious switching activities from other sources in the environment that superpose like crosstalk. These samples have been extracted from several localization cycles and labeled by a human supervisor. Figure 10 shows in the top strip the sampled data of one localization cycle. In the learning phase, "syncrawl" was split into two parts by holdout sampling method resulting in two subdata sets with similar class distribution and data size in order to generate the classifier with optimum parameters. The remaining two raw data sets ("syncraw2," "syncraw3") were employed in the testing phase to analyze performance of trained classifier. This gives 66 examples in "edge" class and 2,056 in "no edge" class. The results are shown in Table 3 . The overall classification rates of SVM-C and the heuristic method are $98.822 \%$ and $97.88 \%$, respectively. But these results look a bit too optimistic, as just the "no edge" events are ruled out well, while the "edge" events still lack a comprehensive number of correct classifications or detections, which means that synchronization cycles occasionally might be missed. However, this is not a major problem, as the system does not need synchronization for each localization cycle. Nevertheless, improvement of this classification subsystem is aspired and is underway.

Figure 10 shows an excerpt from "syncraw2" and "syncraw3" data of the length of one localization cycle in the upper strip and the edge detections of SVM-C versus heuristic method as well as the coil switching control signal time points in the lower strip. The visible constant lag between coil switching control signal and the observed actual edge locations is due to the delay in the currently used power electronics for coil driving. Obviously, the SVM-C solution in the given straight form can already be dealing with noise, spurious switching activities, or crosstalk as well as coils in various distances, while the heuristics fails to do so in a significantly higher number of cases.

Future work has to tackle performance increase along with effort reduction with regard to energy consumption, for example, reducing sampling rate and/or window size. The number of support vectors currently employed is currently computed as 907 with 2,000 features or dimensions each. Possible benefits of feature computation and sequential approaches [36] as well as larger data sets should be regarded for a lean and efficient embedded implementation in followup work. 


\section{Neural Virtual Sensors}

Virtual sensors are an established engineering concept to obtain the equivalent of sensory registration that is not directly amenable to measurement either due to lack of physical transduction principle or due to too expensive available physical transduction principle. A well known example of the latter case is knock-detection in combustion engines, where available but prohibitively expensive pressure sensing is replaced by a feasible acoustical sensing principle [37]. The implied, often nonlinear, mapping task can be well implemented by suitable artificial neural networks, such as, for example, Multi-Layer-Perceptron with Backpropagation learning (MLP), Fahlman's Cascade Correlation (CC) network, Radial-Basis-Function (RBF) networks, or SupportVector-Regression (SVR) networks [37, 38].

3.1. Motivation. In this paper, the most promising neural network candidates, for example, RBF and SVR networks, are investigated as neural virtual sensors to improve localization quality. The basic idea of the localization process including standard method from Section 2 and two different enhancing approaches with neural virtual sensors are illustrated in Figure 11. Two main lines of investigation with the supervised neural virtual sensor approach are depicted by two branches in the figure. The first one employs the model estimated distances as input variables and remaps these to new corrected distances, followed by the standard localization algorithms of Section 2 for coordinate calculation. This method, which requires the actual coil and sensor positions for ground truth distance calculation, will be denoted as the distanceto-distance (D2D) approach. The second one directly maps the acquired sensor voltages to the sensor coordinates or position, completely omitting any model as well as omitting standard localization algorithms. This will be denoted as as the voltage-to-coordinates (V2C) approach. In both cases, representative training data must be provided for the supervised mapping generation in the neural virtual sensors.

The motivation of the proposed approach and its two variations comes from the well known weakness of distance estimation as expressed in (5) and (6). The employed model assumes the sensor to be situated on the principal axis of the respective coil, an assumption that is rarely met in actual sensor locations in container volumes. This implies that the stronger the sensor position deviates from the principal axis of the regarded coil, the larger the resulting error of the estimated distance from the sensor to the corresponding coil will be. Figure 12 illustrates this effect for one $z$-plane of the ISE demonstrator. The error in the center is quite small because the sensor comes closest to the principal coil axes due to the cylindrical arrangement.

The effect, underlying the illustration in Figure 12, is well known and algorithmic correction schemes have long been suggested $[8,9]$. The advantage of the suggested supervised learning approach is that also a calibration of the localization system with regard to instance specifics is achieved. In the referred to patents, also the straight estimation of the sensor location from magnetic sensor readings has been investigated by look-up-table (LUT) mechanisms. The advantages of RBF or SVR approaches with regard to LUT in size, generalization, and so forth are well known and obvious.

3.2. RBF Networks. Regarded RBF networks and tool implementations, in particular, differ in determination mechanism and size of the hidden layer and choice of the employed kernel function, for example, the Gaussian function

$$
h_{i}(x)=\exp \left(-\frac{\left\|x-\mu_{i}\right\|^{2}}{2 \sigma_{i}^{2}}\right),
$$

where $i$ is the index of the hidden layer, $\mu_{i}$ is the center of the corresponding basis function, and $\sigma_{i}$ is the spread which determines the sensitivity of the neuron. The output layer then performs a linear transformation of the hidden neurons activations to the target output values. It is calculated as

$$
f(x)=\sum_{i=1}^{k} w_{i} h_{i}(x)+w_{0}
$$

with $w_{i}$ and $w_{0}$ being the weights. The centers $\mu_{i}$ are learned form the training set and the weights are optimized while training $[39,40]$. In this work, the implementation from MATLAB with the parameters spread and performance goal is employed. A more resource efficient version of the RBF is Platt's Resource-Allocating (RAN) Network for Function Interpolation [41]. RAN allows the growth of the hidden layer from scratch and spread of every kernel function to be adjusted during training [41] and can be for future leaner realizations.

3.3. SVM Regression. Support vector regression (SVR) [42] is an extension of the well established Support-VectorMachines (SVMs) in order to solve the regression problem of learning and predicting continuous domain data. SVR generates models from the training set $\left\{\left(\mathbf{x}^{l}, y^{1}\right), \ldots,\left(\mathbf{x}^{l}, y^{l}\right)\right\}$ that perform with best fit in a linear function $f(\mathbf{x})=$ $\langle\mathbf{w}, \mathbf{x}\rangle+b$ and result with a minimum $\epsilon$ deviation in the loss function. Using $\epsilon$-insensitive loss function to reduce the error to zero for all points that are smaller than $\epsilon$, in some training points, however, this error is beyond $\epsilon$; to deal with unfeasible constraints, the slack variable $\xi$ is introduced in the optimization problem. The optimization problem of $\epsilon$ insensitive support vector regression ( $\epsilon$-SVR) [42] can be formulated as

$$
\begin{array}{cc}
\operatorname{minimize} & \frac{1}{2}\|\mathbf{w}\|^{2}+C \sum_{i=1}^{l}\left(\xi_{i}+\xi_{i}^{*}\right) \\
\text { subject to } & y_{i}-\left\langle\mathbf{w}, \mathbf{x}_{\mathbf{i}}\right\rangle-b \leq \epsilon+\xi_{i}, \\
& \left\langle\mathbf{w}, \mathbf{x}_{\mathbf{i}}\right\rangle+b-y_{i} \leq \epsilon+\xi_{i}^{*}, \\
& \xi_{i}, \xi_{i}^{*} \geq 0, \quad i=1,2, \ldots l,
\end{array}
$$

where $C$ determines the trade-off between the model complexity and the tolerance of the deviations larger than $\epsilon$. The regression function is given by transforming the problem in 
(13) into its dual problem subject to $0<\alpha_{i}$, $\alpha_{i}^{*}<C$ and $\sum_{i=1}^{l}\left(\alpha_{i}-\alpha_{i}^{*}\right)=0$

$$
f(\mathbf{x})=\sum_{i, j=1}^{n_{S V}}\left(\alpha_{i}-\alpha_{i}^{*}\right) K\left(\mathbf{x}_{\mathbf{i}}, \mathbf{x}_{\mathbf{j}}\right)+b,
$$

where $n_{\mathrm{SV}}$ is the number of support vectors (SVs) and $\alpha_{i}$ and $\alpha_{i}^{*}$ are Lagrangian multipliers. The kernel function $K\left(\mathbf{x}_{\mathbf{i}}, \mathbf{x}_{\mathbf{j}}\right)=\Phi\left(\mathbf{x}_{\mathbf{i}}\right) \cdot \Phi\left(\mathbf{x}_{\mathbf{j}}\right)$ can be chosen as radial basis function (RBF). Applying the so-called kernel trick allows tackling of a nonlinear regression problem with linear estimation by mapping the data set into a higher dimensional space. The $\mathrm{RBF}$ kernel function is computed as

$$
K\left(\mathbf{x}_{\mathbf{i}}, \mathbf{x}_{\mathbf{j}}\right)=\exp \left(-\gamma\left\|\mathbf{x}_{\mathbf{i}}-\mathbf{x}_{\mathbf{j}}\right\|^{2}\right), \quad \gamma>0
$$

The optimum generalization performance of SVR is based on the setting of model parameters $\epsilon$ which is usually assigned as level of typical noise in the training data, as well as parameter $C$, and the kernel parameter $\gamma$. For finding a convergence point of the optimum SVR prediction performance, a gridsearch method is commonly suggested [43], as independent characteristics to prediction model of $C$ and $\gamma$.

\section{Experiments and Results}

The data sets introduced in Section 2.4 will serve now for experimental validation according to the outline in Figure 11 of the proposed methods. For this aim, the data sets have to be sampled to generate appropriate training and test sets for the supervised learning of the neural virtual sensors. With regard to the moderate but sufficient available data size, the holdout approach was adopted. Table 4 summarizes the selected training sets. The residual data of each demonstrator are saved as test sets.

For the ISEL $\mathrm{I}_{1}$ data set the measured points are orthogonally located in one $z$-plane which can be seen in Figure 12. The training data contains 25 input-target pairs which are marked by the filled circles in the corresponding following error maps (Figures 14 and 16). The BREW data set positions are spatially less regularly distributed (see Figure 8). Every position with even index is used for training and the positions with odd index are used for test set, resulting in a training data set size of 165 trials and a test data set size of 160 trials. For the HMI data set, there are 44 different positions, whereas at each height ( $z$-position) 4 different $x$ - $y$-positions where acquired. This results in $11 z$-planes of $4 x$-y-positions each. The training data set is composed of $6 z$-planes and the test set contains the remaining $5 z$-planes, whereby test and train $z$-planes alternate.

For D2D remapping networks, which correspond to path 2 in Figure 11, two different network architectures with suitable parameter setting ranges have been investigated, based on a standard MATLAB implementation. The varied parameters are the spread $(\sigma)$ and the performance goal, which is defined as the mean squared error of the training data. The architecture examined first is a network with input and output layer size equal to the number of coils. With
TABLE 4: Training data sets.

\begin{tabular}{lccc}
\hline & $\begin{array}{c}\text { ISEL } \\
\text { train set }\end{array}$ & $\begin{array}{c}\text { BREW } \\
\text { train set }\end{array}$ & $\begin{array}{c}\text { HMI } \\
\text { train set }\end{array}$ \\
\hline Number of positions & 169 & 30 & 44 \\
Number of trials per position & 1 & min. 10 & 3 \\
Total number of trials & 169 & 325 & 132 \\
Number of trained positions & 25 & 15 & 24 \\
Number of trained trials & 25 & 165 & 72 \\
\hline
\end{tabular}

$M$ being the number of coils and $N$ being the number of hidden layer neurons, the network architecture can be referred as $M x N_{i} x M$. The second architecture consists of $M$ individually trained networks of $M x N_{i} x 1$ topology. The number of networks grows linearly with the number of coils and can be more greedy with regard to resources, but hidden layers can be individually grown, with some similarity to RAN [41], and convergence commonly is easier. This architecture will be denoted as $M x\left(M x N_{i} x 1\right)$ in the following.

For V2C mapping the same approach will be pursued. However, in the case of the multinetwork architecture only three coordinates have to be generated, independent of the number of coils. So the architecture for V2C mapping and one single net is $M x N \times 3$ and for multiple networks it is $3 x\left(3 x M x N_{i} x 1\right)$, obviously alleviating resource issues and the training process. The $\mathrm{V} 2 \mathrm{C}$ approach is illustrated by path 3 in Figure 11. All the presented results are achieved using the multiple network architectures for both D2D and V2C.

For determining an optimum RBF parameter set, a basic sensitivity analysis has been carried out with regard to mean localization error minimization and generalization maximization. The investigated RBF parameters are the performance goal and the spread. First, the performance goal is set to fixed values of either $1,0.1$, or 0.01 to limit the effort to a one-dimensional search. For these three different settings the spread is swept. With this approach a local, suitable optimum combination of performance goal and spread quality can be achieved, which returns a minimized distance error and hence localization error. Figure 13 shows one example of a spread sweep for the BREW data set and D2D remapping. The localization error is computed for either the entire data set (training + test data set) and for the test data set. The optimum spread settings for those two data sets and analysis runs are not identical. Currently, the RBF spread which performs best for the test set is chosen. This approach can be applied for all network architectures for D2D remapping and for V2C, except for D2D with multiple network architecture case. The spread is swept for each network individually to make sure that an optimum spread can be found for each coil. If the criteria would be the localization error, there would be no way to extract the best spreads because the multilateration performs a transformation from an $\mathrm{M}$-dimensional input to the 3-dimensional output. So, in case of D2D remapping with $M x\left(M x N_{i} x 1\right)$ architecture the criteria are the distance error which can be calculated before computing multilateration. 
TABLE 5: Results for raw data using advanced algorithms as described in Section 2.3. The parameter settings can be found in Figure 6. The results are a mean of five runs.

\begin{tabular}{lcccccccccccc}
\hline \multirow{2}{*}{ Error } & \multicolumn{4}{c}{ Raw brew data } & \multicolumn{9}{c}{ Raw ISEL data } & \multicolumn{4}{c}{ Raw HMI Data } \\
& GD & SA & PSO & ML & GD & SA & PSO & ML & GD & SA & PSO & ML \\
\hline \multirow{2}{*}{ Loc. error $\mu$} & 8.81 & $\mathbf{8 . 6 7}$ & 8.7 & 13.18 & 2.92 & 2.89 & $\mathbf{2 . 8 8}$ & 3.65 & 10.01 & 10.06 & $\mathbf{9 . 8 5}$ & 11.81 \\
$\%$ & 2.37 & 2.33 & 2.34 & 3.54 & 1.05 & 1.04 & 1.04 & 1.32 & 16.41 & 16.49 & 16.15 & 19.36 \\
Loc. error $\sigma$ & 4.17 & 4.17 & $\mathbf{4 . 1 5}$ & 11.97 & 1.9 & 1.94 & $\mathbf{1 . 8 8}$ & 2.2 & 9.5 & $\mathbf{9 . 4 6}$ & 9.56 & 12.8 \\
\% & 1.12 & 1.12 & 1.12 & 3.22 & 0.69 & 0.7 & 0.68 & 0.79 & 15.57 & 15.51 & 15.67 & 20.98 \\
Max. loc. error & 35.5 & 37.05 & $\mathbf{3 5 . 3 4}$ & 135.66 & $\mathbf{1 1 . 1 5}$ & 11.57 & 11.31 & 14.45 & 67.45 & 67.02 & $\mathbf{6 7 . 3 7}$ & 137.25 \\
$\%$ & 9.54 & 9.96 & 9.5 & 36.47 & 4.03 & 4.18 & 4.08 & 5.22 & 110.57 & 109.87 & 110.44 & 225.0 \\
\hline
\end{tabular}

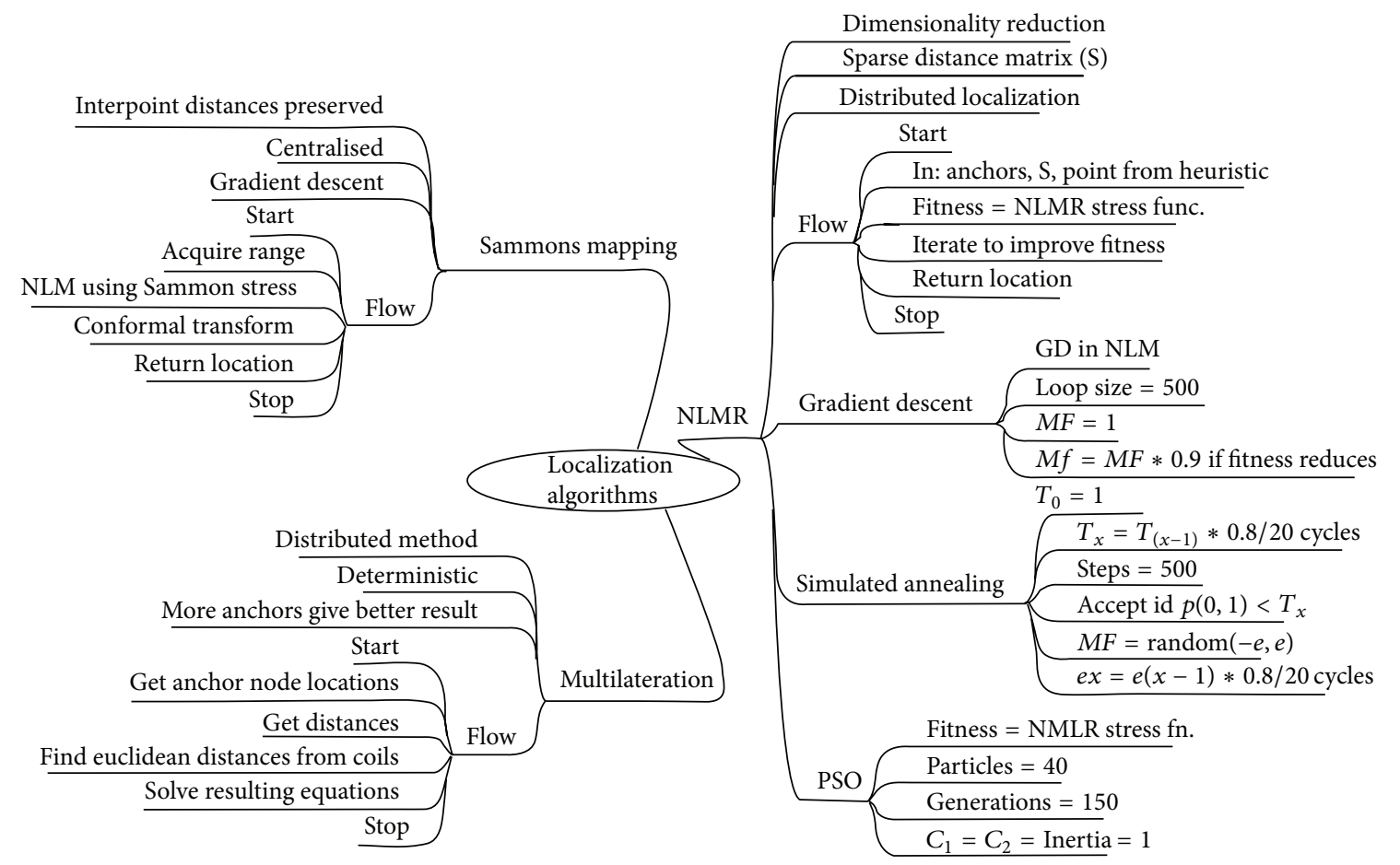

FIGURE 6: Survey of employed algorithms and corresponding parameter settings.

For each coil, there is a specific RBF spread which results in a minimum distance error.

SVR is employed as the second method in the entire experiments with identical train and test data sets to RBF part of the work. Here the LIBSVM [44] library was implemented on MATLAB platform. Input and supervised learning data for D2D and V2C investigations were identical to the RBF case, too. Applying a grid search method, to cover a wide spectrum of parameter space in searching, model parameters $C$ and $\gamma$ are determined in the range of $[1,100,000]$ and $[0.1,100]$, respectively. Parameter $\epsilon$ is usually defined to the level of typical noise in the training data. In the training phase, the pair of parameters $C$ and $\gamma$ delivering the minimal mean square error of the model validation process will be selected to generate the prediction model. The particular setting values of $\varepsilon$ for the ISEL1, BREW, and HMI data are $0.03,0.01$, and 0.04 for D2D and $0.01,0.01$, and 0.03 for $\mathrm{V} 2 \mathrm{C}$, respectively.
The outlined experiments are conducted for each data set with RBF and SVM, each performing D2D and V2C mapping. Each best performing network is trained and recalled at least 3 times to make sure that random initialization effects do not affect the results. The results are presented in the following two subsections.

To put the upcoming results and improvements into perspective, in addition to standard multilateration, we have applied the advanced methods from Section 2.3 to all three raw, $\alpha$-corrected distance data sets (see (6)). The achieved localization quality is shown in Table 5 , which shows substantial improvements to multilateration for all methods, but, in particular, for the PSO based method.

4.1. Distance to Distance. The $\mathrm{ISEL}_{1}$ data set has a mean localization error of $22.80 \mathrm{~cm}$ and a maximum localization error of $41.27 \mathrm{~cm}$ applying standard multilateration. By setting the coil distance scale factor $\alpha$ to its optimum value of 1.35 , the mean 
AMR sensor RAW data of $X$-axis
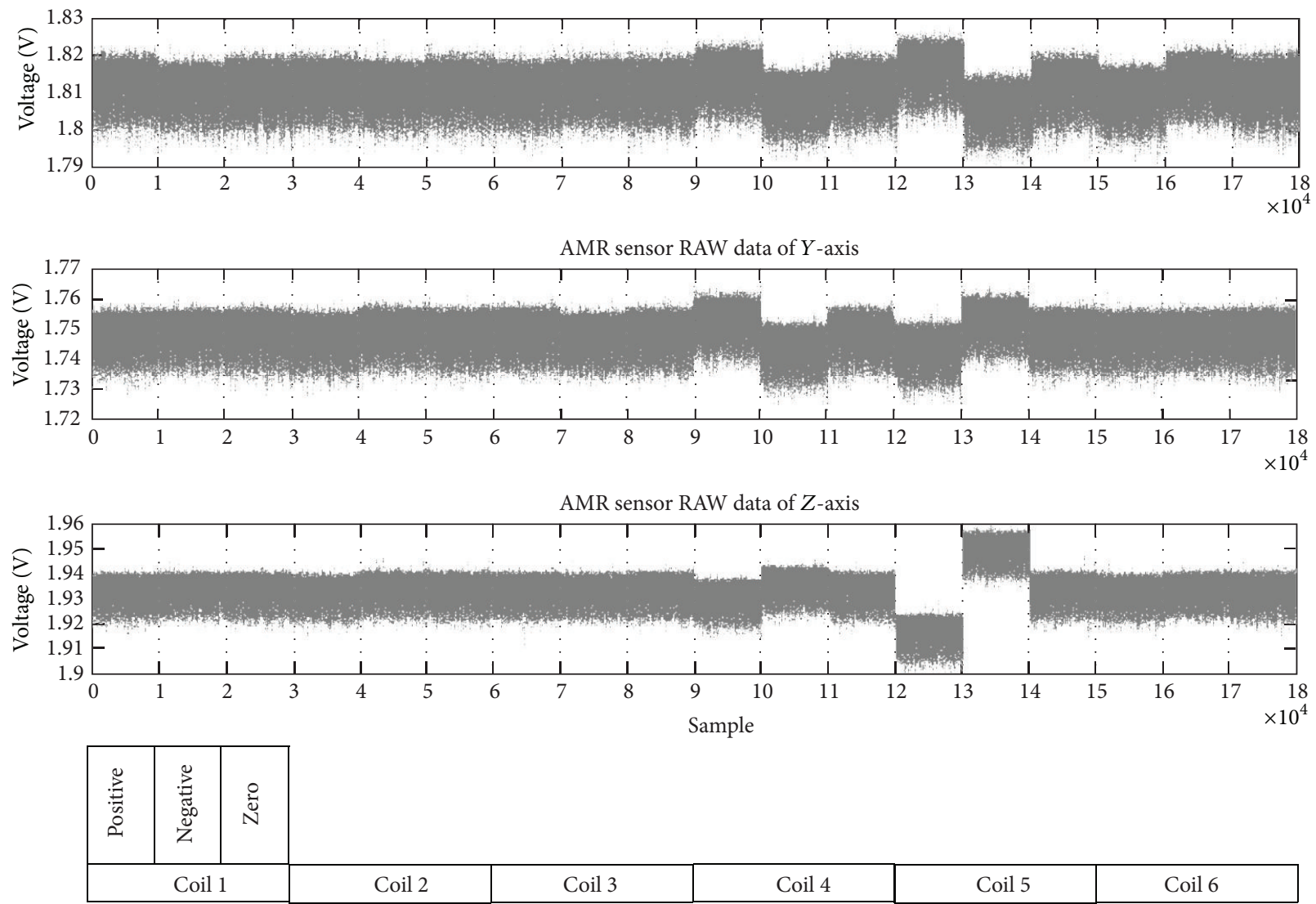

FIGURE 7: 3D-AMR-sensor raw data sketch from ISEL 1 data set for a six coil cycle.

Ground truth positions measured at Technikum Warsteiner

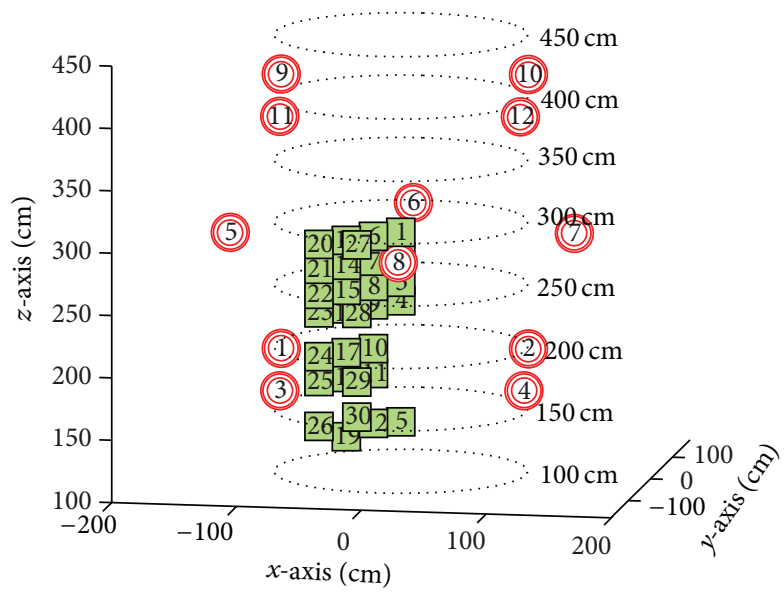

FIGURE 8: The 30 positions measured for the brewery data set are visualized here. The ground truth positions of the sensor are marked by the rectangles, the circles determine the positions of the 12 coils.

error can be reduced to $3.60 \mathrm{~cm}$ and the maximum error is reduced to $15.03 \mathrm{~cm}$. The $\mathrm{D} 2 \mathrm{D}$ remapping approach applied to the ISEL $\mathrm{I}_{1}$ data set leads to a further improvement. The error map in Figure 14 shows that the maximum localization error is reduced by a factor of 8 compared to the initial results of
Figure 12 which are achieved without any scaling factor or neural virtual sensor. The mean error is reduced by a factor of 21 to just $1.05 \mathrm{~cm}$ for the test data set.

Table 6 summarizes the results for RBF and SVR in D2D mapping of ISEL $L_{1}$ data. The two networks are compared side by side for each of the data sets.

Without D2D remapping, the mean localization error for the BREW data set is $13.18 \mathrm{~cm}$ and the maximum error is $135.66 \mathrm{~cm}$. By comparing the mean localization errors of both approaches for the test sets from Table 6, it can be seen that the RBF generalizes better than the SVR.

The mean localization without D2D remapping for the HMI data set is $11.75 \mathrm{~cm}$ and maximum error is $121.61 \mathrm{~cm}$. Compared to the actual demonstrator dimensions (see Table 1) which is the smallest of all three demonstrators the initial error for the standard method (multilateration without D2D remapping) is very high. This is due to the use of the built-in ADC of the $\mu \mathrm{C}$ which has a resolution of 12 bit compared to 16 bit of the DAQ and a different sensor which is less sensitive than the one used with the first two data sets. Both methods improve the localization result. See Table 6 for more details.

To better assess these results and improvements, in addition to standard multilateration, we have applied the advanced methods from Section 2.3 to all three $\alpha$-corrected, D2D remapped distance data sets. This has been done for 


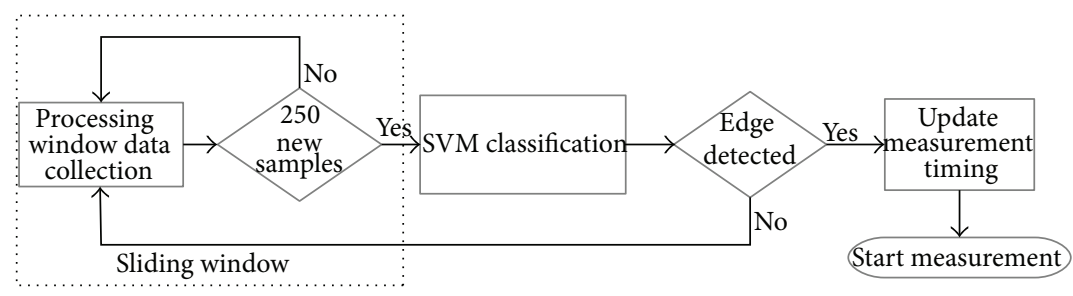

FIGURE 9: Processing structure of SVM based edged detection system.
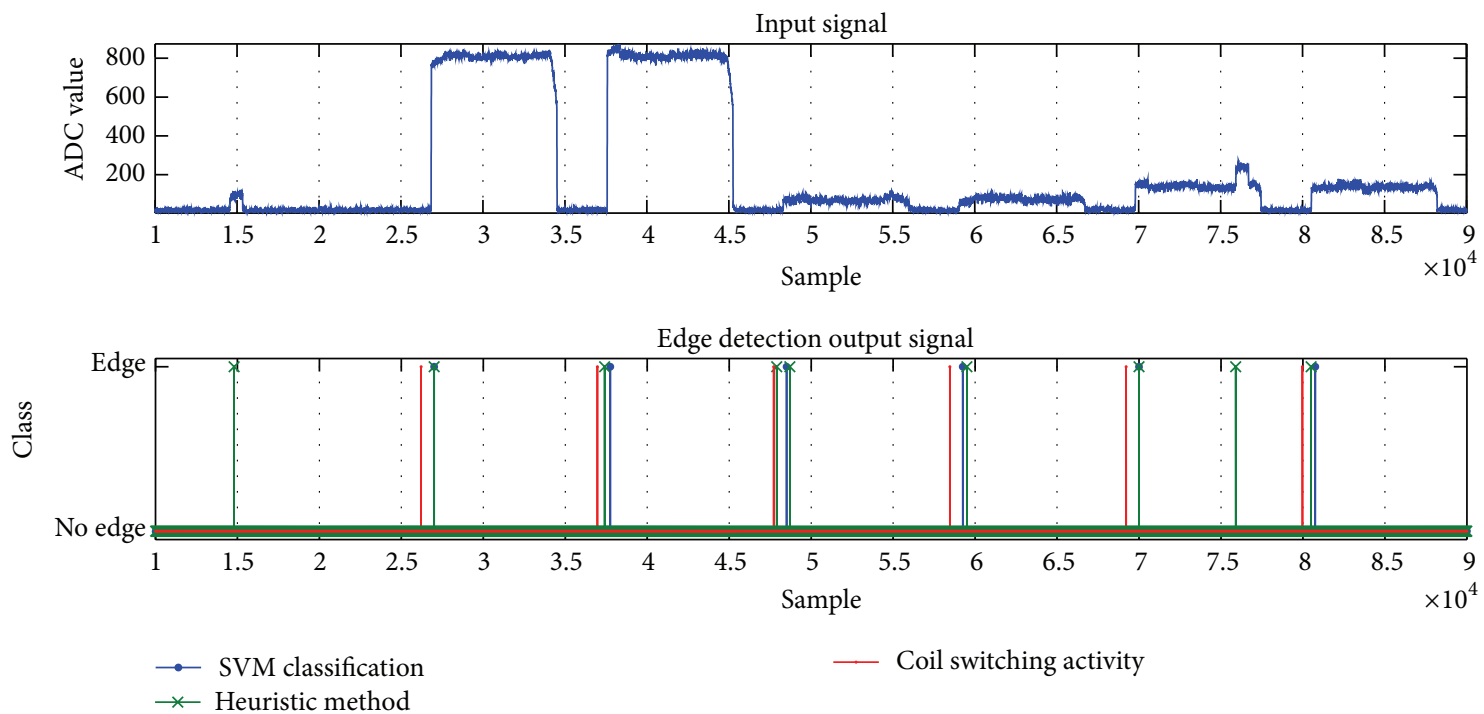

FIGURE 10: Edge detection result based on SVM classification for magnetic synchronization: top strip shows exemplary raw data from complete localization data from 3D-AMR-sensor and 12-bit ADC of $\mu \mathrm{C}$, and bottom strip shows edge detection times of SVM and heuristic method as well as coil switching control signal for the top data.

(1) Basic approach with error prone $B$-field model and localization algorithm

(2) Distance-2-distance remapping to correct distance error

(3) Voltage-2-coordinates mapping to bypass distance calculationi and localization algorithm

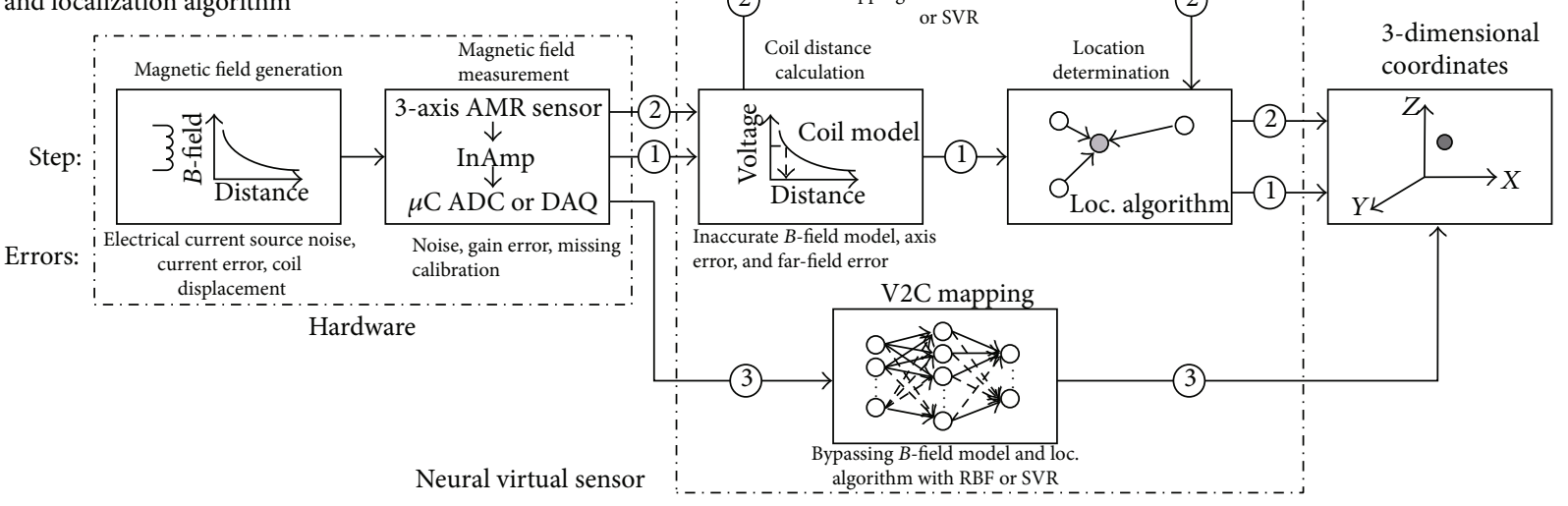

FIGURE 11: The three different methods of determining the position of the sensor are illustrated here. The first approach is straight forward, whereas the second method minimizes the distance error by remapping the distances before coordinate determination. Sensor voltages are directly mapped to coordinates with the third method to bypass model-based distance calculation and the localization algorithm. 


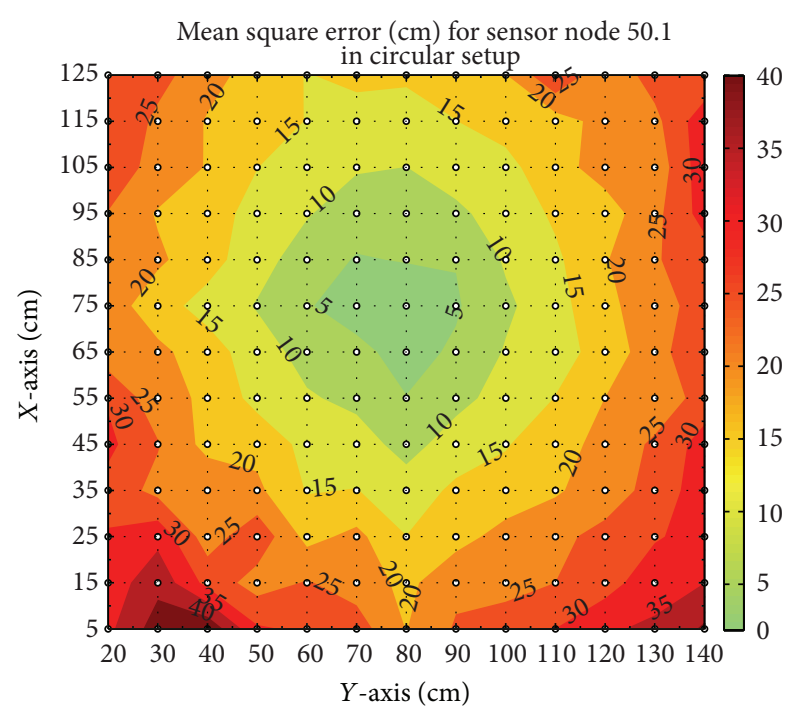

- Mean square error $(\mathrm{cm})$

- Sample point

FIGURE 12: Error map for ISE demonstrator and using simple multilateration. The localization error increases with increasing distance to the center of the volume.

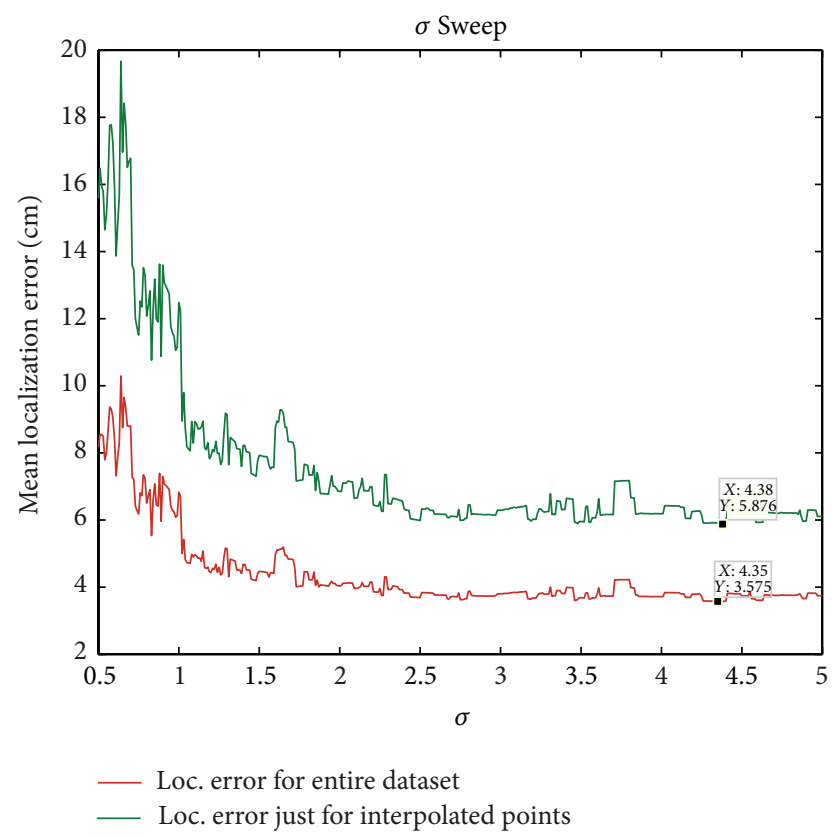

FIgURE 13: Dependent on the RBF spread the resulting localization error varies and a minimum can be determined.

RBF and SVR for complete data sets as well as test data only (Figure 15). The achieved localization quality is given in Table 7, which again shows substantial improvements both to the results before D2D remapping as well as to standard multilateration application for all methods. This has been summarized in Figure 15. As before, the PSO based method is taking the lead in result quality.

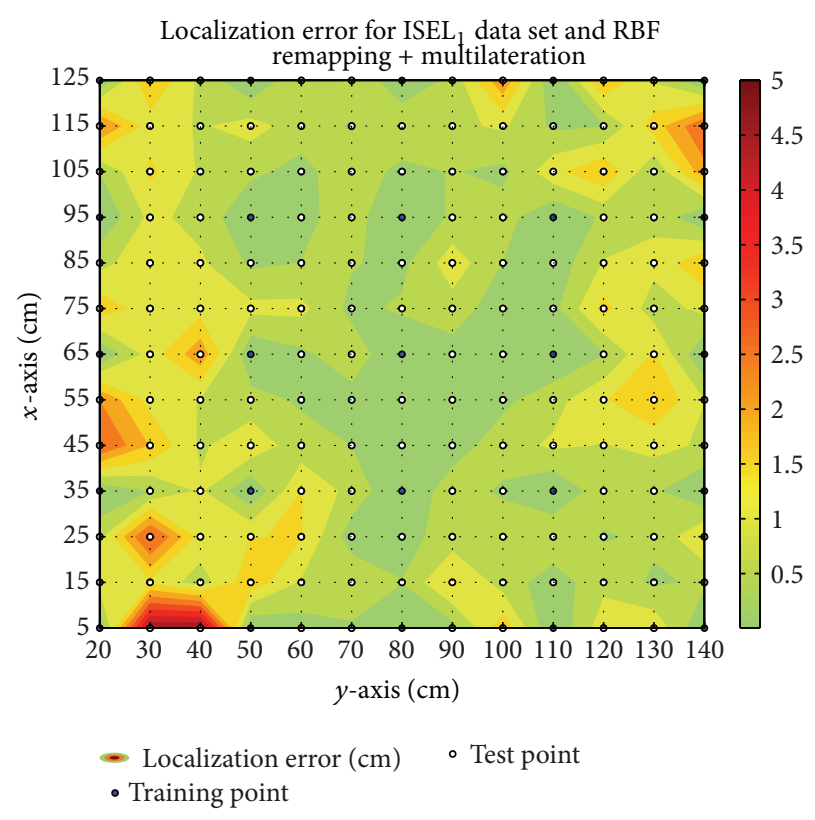

FIGURE 14: Employing RBF-D2D remapping and multilateration the mean localization error can be reduced to $0.90 \mathrm{~cm}$.

TABLE 6: Results for all experiments employing D2D and ML computation for path 2 in Figure 11.

\begin{tabular}{lcccccc}
\hline Data set & \multicolumn{2}{c}{ ISEL $_{1}$} & \multicolumn{2}{c}{ BREW } & \multicolumn{2}{c}{ HMI } \\
Network type & RBF & SVR & RBF & SVR & RBF & SVR \\
\hline$\sum N_{i}$ & 139 & 150 & 333 & 492 & 162 & 216 \\
\hline \multicolumn{5}{c}{ Results for test and training data set in $[\mathrm{cm}]$} \\
Loc. error $\mu$ & 0.90 & $\mathbf{0 . 8 4}$ & 3.52 & $\mathbf{3 . 5 1}$ & 2.86 & $\mathbf{2 . 2 1}$ \\
\% & 0.32 & 0.3 & 0.95 & 0.94 & 4.69 & 3.62 \\
Loc. error $\sigma$ & 0.80 & $\mathbf{0 . 6 5}$ & $\mathbf{4 . 1 3}$ & 4.47 & 3.46 & $\mathbf{3 . 3 0}$ \\
\% & 0.29 & 0.23 & 1.11 & 1.2 & 5.67 & 5.41 \\
Max. loc. error & 5.04 & $\mathbf{3 . 1 3}$ & 37.04 & $\mathbf{3 5 . 6 2}$ & $\mathbf{2 1 . 4 1}$ & 21.41 \\
\% & 1.82 & 1.13 & 9.96 & 9.58 & 35.1 & 35.1 \\
\hline \multicolumn{7}{c}{ Results for test with test data set in [cm] } \\
\%oc. error $\mu$ & 1.05 & $\mathbf{0 . 9 1}$ & $\mathbf{5 . 6 0}$ & 6.24 & 4.84 & $\mathbf{4 . 3 6}$ \\
Loc. error $\sigma$ & 0.38 & 0.33 & 1.51 & 1.68 & 7.93 & 7.15 \\
\% & 0.78 & $\mathbf{0 . 6 4}$ & 5.01 & $\mathbf{4 . 9 9}$ & 4.26 & $\mathbf{3 . 8 2}$ \\
Max. loc. error & 0.28 & 0.23 & 1.35 & 1.34 & 6.98 & 6.26 \\
\% & 5.04 & $\mathbf{3 . 1 3}$ & 37.04 & $\mathbf{3 5 . 6 2}$ & $\mathbf{2 1 . 4 1}$ & 21.41 \\
\hline
\end{tabular}

4.2. Voltage to Coordinate. The results for V2C mapping approach are found to be comparable in terms of localization error to the previous approach. Figure 16 shows the error map for RBF-V2C applied to ISEL ${ }_{1}$ data set. The mean localization error for $\mathrm{ISEL}_{1}$ data set is higher than the one of D2D followed by standard multilateration but still is in an acceptable range of just $2.14 \mathrm{~cm}$, which is in the order of the current sensor's dimensions and, thus, sufficient for the regarded application. Table 8 summarizes and compares RBF and SVR for V2C in the same way as previously provided for the D2D investigations. The SVR approach for ISEL $\mathrm{L}_{1}$ data 
TABLE 7: Results after D2D mapping using advanced algorithms as described in Section 2.3. The parameter settings can be found in Figure 6. The results are a mean of five runs.

\begin{tabular}{|c|c|c|c|c|c|c|c|c|c|c|c|c|}
\hline \multirow{2}{*}{ Error } & \multicolumn{4}{|c|}{ Brew data } & \multicolumn{4}{|c|}{ ISEL $_{1}$ data } & \multicolumn{4}{|c|}{ HMI data } \\
\hline & GD & SA & PSO & ML & GD & SA & PSO & ML & GD & SA & PSO & ML \\
\hline \multicolumn{13}{|c|}{ Results for D2D [Rbf] train + test data set in $[\mathrm{cm}]$} \\
\hline Loc. error $\mu$ & 3.72 & 4.02 & 3.2 & 3.52 & 0.92 & 0.94 & 0.92 & 0.9 & 3.0 & 2.96 & 2.67 & 2.86 \\
\hline$\%$ & 1.0 & 1.08 & 0.86 & 0.95 & 0.33 & 0.34 & 0.33 & 0.32 & 4.92 & 4.85 & 4.38 & 4.69 \\
\hline Loc. error $\sigma$ & 3.1 & 3.01 & 3.25 & 4.13 & 0.7 & 0.66 & 0.67 & 0.8 & 3.33 & 3.22 & 3.27 & 3.44 \\
\hline$\%$ & 0.83 & 0.81 & 0.87 & 1.11 & 0.25 & 0.24 & 0.24 & 0.29 & 5.46 & 5.28 & 5.36 & 5.64 \\
\hline Loc. error max & 18.71 & 18.73 & 18.98 & 37.04 & 3.83 & 3.89 & 3.97 & 5.04 & 20.33 & 20.65 & 20.32 & 21.18 \\
\hline$\%$ & 5.03 & 5.03 & 5.1 & 9.96 & 1.38 & 1.4 & 1.43 & 1.82 & 33.33 & 33.85 & 33.31 & 34.72 \\
\hline \multicolumn{13}{|c|}{ Results for D2D [Rbf] test data set in $[\mathrm{cm}]$} \\
\hline Loc. error $\mu$ & 5.15 & 5.32 & 4.73 & 5.14 & 1.58 & 1.58 & 1.61 & 1.76 & 4.6 & 4.59 & 4.27 & 4.54 \\
\hline$\%$ & 1.38 & 1.43 & 1.27 & 1.38 & 0.57 & 0.57 & 0.58 & 0.64 & 7.54 & 7.52 & 7.0 & 7.44 \\
\hline Loc. error $\sigma$ & 3.54 & 3.51 & 3.74 & 4.35 & 0.93 & 0.94 & 0.89 & 1.2 & 4.21 & 4.06 & 4.15 & 4.29 \\
\hline$\%$ & 0.95 & 0.94 & 1.01 & 1.17 & 0.34 & 0.34 & 0.32 & 0.43 & 6.9 & 6.66 & 6.8 & 7.03 \\
\hline Loc. error max & 18.71 & 18.73 & 18.98 & 32.15 & 3.67 & 3.73 & 3.76 & 4.94 & 20.33 & 20.65 & 20.32 & 21.18 \\
\hline$\%$ & 5.03 & 5.03 & 5.1 & 8.64 & 1.32 & 1.35 & 1.36 & 1.78 & 33.33 & 33.85 & 33.31 & 34.72 \\
\hline \multicolumn{13}{|c|}{ Results for D2D [SVR] train + test data set in [cm] } \\
\hline Loc. error $\mu$ & 3.73 & 3.85 & 3.01 & 3.51 & 0.8 & 0.89 & 0.76 & 0.84 & 2.42 & 2.48 & 2.28 & 2.21 \\
\hline$\%$ & 1.0 & 1.03 & 0.81 & 0.94 & 0.29 & 0.32 & 0.27 & 0.3 & 3.97 & 4.07 & 3.74 & 3.62 \\
\hline Loc. error $\sigma$ & 3.2 & 2.99 & 3.41 & 4.46 & 0.52 & 0.52 & 0.51 & 0.65 & 3.14 & 3.13 & 3.25 & 3.29 \\
\hline$\%$ & 0.86 & 0.8 & 0.92 & 1.2 & 0.19 & 0.19 & 0.18 & 0.23 & 5.15 & 5.13 & 5.33 & 5.39 \\
\hline Loc. error max & 17.98 & 18.98 & 18.52 & 35.62 & 2.92 & 2.91 & 3.07 & 3.14 & 20.26 & 20.62 & 20.54 & 21.41 \\
\hline$\%$ & 4.83 & 5.1 & 4.98 & 9.58 & 1.05 & 1.05 & 1.11 & 1.13 & 33.21 & 33.8 & 33.67 & 35.1 \\
\hline \multicolumn{13}{|c|}{ Results for D2D [SVR] test data set in $[\mathrm{cm}]$} \\
\hline Loc. error $\mu$ & 5.26 & 4.97 & 4.76 & 5.61 & 0.78 & 0.89 & 0.74 & 0.81 & 4.19 & 4.25 & 4.18 & 4.22 \\
\hline$\%$ & 1.41 & 1.34 & 1.28 & 1.51 & 0.28 & 0.32 & 0.27 & 0.29 & 6.87 & 6.97 & 6.85 & 6.92 \\
\hline Loc. error $\sigma$ & 3.67 & 3.62 & 3.82 & 4.51 & 0.52 & 0.52 & 0.5 & 0.64 & 3.88 & 3.87 & 3.95 & 3.89 \\
\hline$\%$ & 0.99 & 0.97 & 1.03 & 1.21 & 0.19 & 0.19 & 0.18 & 0.23 & 6.36 & 6.34 & 6.48 & 6.38 \\
\hline Loc. error max & 17.98 & 18.98 & 18.52 & 23.83 & 2.92 & 2.91 & 3.07 & 3.14 & 20.26 & 20.62 & 20.54 & 21.41 \\
\hline$\%$ & 4.83 & 5.1 & 4.98 & 6.41 & 1.05 & 1.05 & 1.11 & 1.13 & 33.21 & 33.8 & 33.67 & 35.1 \\
\hline
\end{tabular}

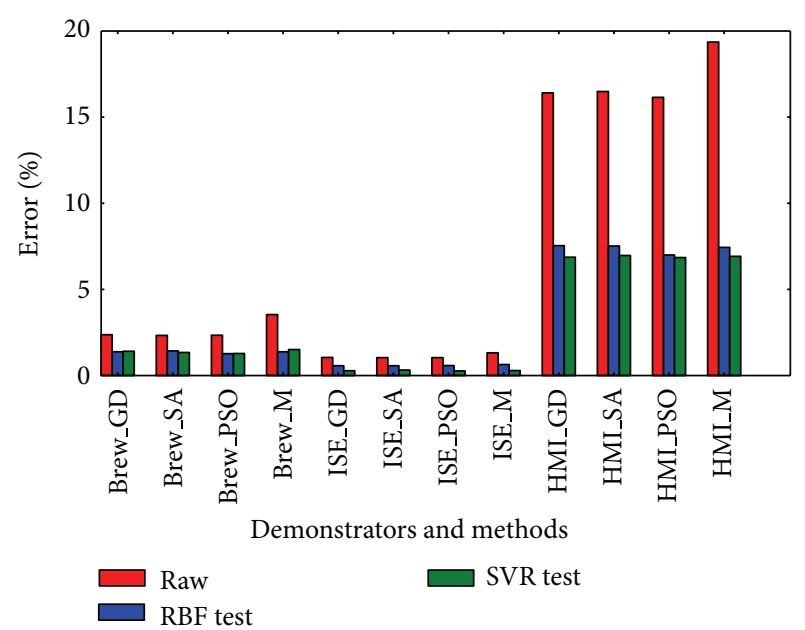

FIGURE 15: Graphical comparison of the raw results with the results after application of RBf and SVR based on Tables 5 and 7. performs superior to the RBF approach, but both approaches lag behind all previously obtained D2D results.

For the BREW data set the mean localization error for $\mathrm{V} 2 \mathrm{C}$ and RBF is nearly twice the one of D2D (RBF) with ML. The maximum error unfortunately is also increased. Nevertheless,one advantage of the $\mathrm{V} 2 \mathrm{C}$ approach is the significantly smaller number of neurons of just 27, while D2D (RBF) and ML required 333 neurons. A trade-off of result quality and computational resource can be considered. The SVR approach for BREW data performs superior to RBF approach but also lags behind $\mathrm{D} 2 \mathrm{D}$ results.

For the HMI data set, an increase in mean localization error can also be observed but is not so expressed as observed for the first two regarded data sets. Comparing the results from Table 8 with those from Table 6 results do not differ significantly, except for the mean localization error for the test data set where D2D (RBF) and ML perform $0.93 \mathrm{~cm}$ better than V2C. However, the SVR V2C approach outperforms 


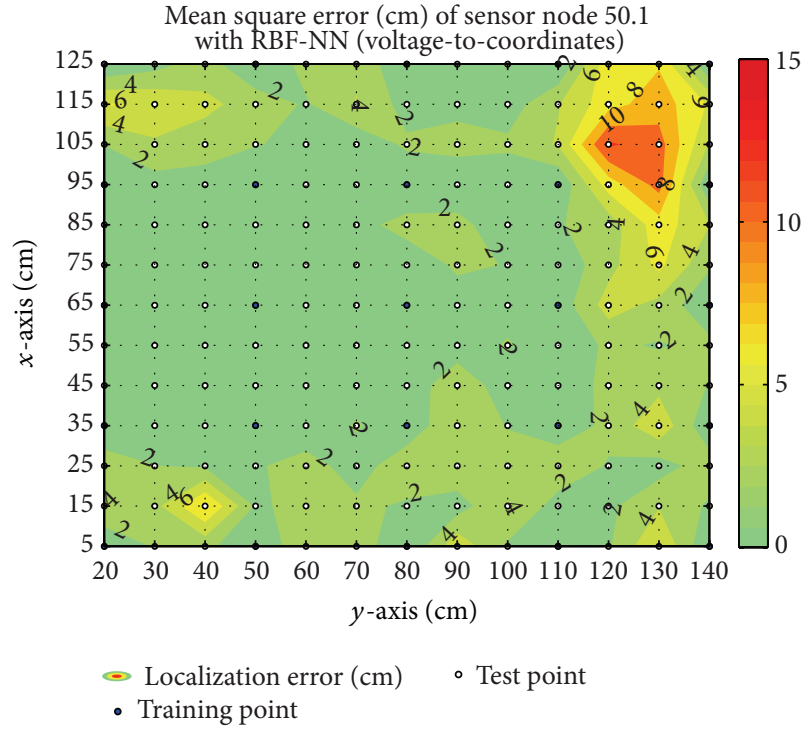

FIGURE 16: The localization error with RBF-V2C mapping is reduced down to $2.14 \mathrm{~cm}$.

TABLE 8: Results for all experiments employing V2C computation for path 3 in Figure 11.

\begin{tabular}{lcccccc}
\hline Data set & \multicolumn{2}{c}{ ISEL $_{1}$} & \multicolumn{2}{c}{ BREW } & \multicolumn{2}{c}{ HMI } \\
Network type & RBF & SVR & RBF & SVR & RBF & SVR \\
\hline$\sum N_{i}$ & 21 & 50 & 27 & 495 & 192 & 213 \\
\hline \multicolumn{6}{c}{ Results for test with test and training data set in [cm] } \\
Loc. error $\mu$ & 2.14 & $\mathbf{1 . 7 8}$ & 7.36 & $\mathbf{4 . 0 4}$ & 2.62 & $\mathbf{1 . 8 1}$ \\
$\%$ & 0.77 & 0.64 & 1.98 & 1.09 & 4.3 & 2.97 \\
Loc. error $\sigma$ & 2.00 & $\mathbf{1 . 6 2}$ & 8.43 & $\mathbf{5 . 4 0}$ & 4.00 & $\mathbf{2 . 5 6}$ \\
\% & 0.72 & 0.58 & 2.27 & 1.45 & 6.56 & 4.2 \\
Max. loc. error & 13.27 & $\mathbf{9 . 2 6}$ & 111.13 & $\mathbf{3 9 . 1 7}$ & 21.10 & $\mathbf{1 6 . 6 9}$ \\
\% & 4.79 & 3.34 & 29.87 & 10.53 & 34.59 & 27.36 \\
\hline \multicolumn{7}{c}{ Results for test with test data set in $[\mathrm{cm}]$} \\
Loc. error $\mu$ & 2.46 & $\mathbf{1 . 9 5}$ & 9.21 & $\mathbf{7 . 1 6}$ & 5.75 & $\mathbf{3 . 5 5}$ \\
\% & 0.89 & 0.7 & 2.48 & 1.92 & 9.43 & 5.82 \\
Loc. error $\sigma$ & 2.01 & $\mathbf{1 . 5 9}$ & 7.20 & $\mathbf{5 . 4 1}$ & 4.14 & $\mathbf{2 . 8 2}$ \\
\% & 0.73 & 0.57 & 1.94 & 1.45 & 6.79 & 4.62 \\
Max. loc. error & 13.27 & $\mathbf{9 . 2 6}$ & 51.58 & $\mathbf{3 3 . 0 7}$ & 21.40 & $\mathbf{1 6 . 6 9}$ \\
\% & 4.79 & 3.34 & 13.87 & 8.89 & 35.08 & 27.36 \\
\hline
\end{tabular}

RBF-based methods and D2D approaches in general for HMI test data.

4.3. Discussion. The overall results given in Tables 6 and 8 show that all proposed methods are feasible and provide substantial result improvements with regard to raw model output data and standard multilateration. The methods from Section 2.3 are already powerful and completely unsupervised. However, they have to rely on the model output and offer no calibration options. The D2D extension, in particular when combined with the methods from Section 2.3, offers calibration options and remarkable result improvement at substantial computational cost, dependence on the model for distance calculation, and the necessity to obtain

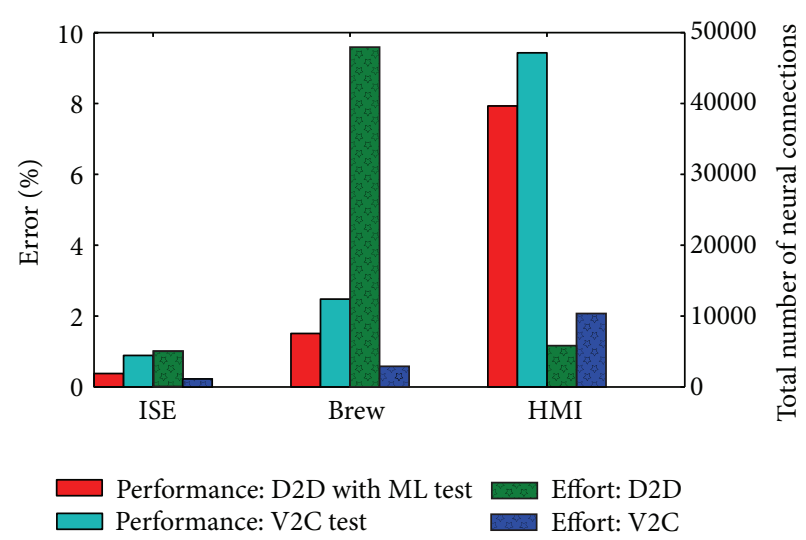

FIGURE 17: Comparison of performance versus effort for D2D and V2C approaches employing RBF neural network (see Tables 6 and $8)$.

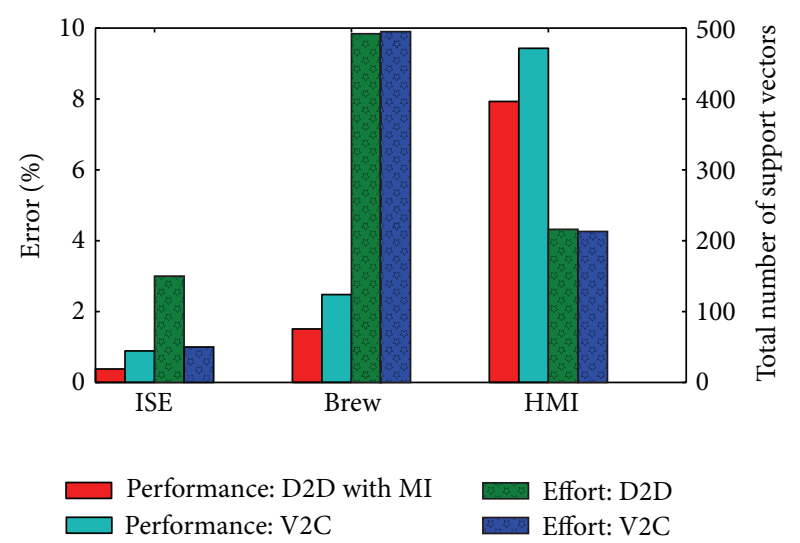

FIGURE 18: Comparison of performance versus effort for D2D and V2C approaches employing SVR (see Tables 6 and 8).

representative supervised data for each application scenario. These methods are best sited for host-based, postmeasurement localization computation.

The V2C approach, though it did not give top performance in most of the investigations, still is appealing, as it is not depending on a model and is computationally much less demanding, because $\mathrm{V} 2 \mathrm{C}$ is linear in complexity with regard to the number of coils and requires smaller network sizes (see first row of Table 6 and of Table 8, resp.). Thus, it is better suited for on-line node-based localization computation and offers calibration options with the same need met in D2D of obtaining representative supervised data for each application scenario. Also, sometimes mediocre results of V2C are partly due to lack of rotation invariance with regard of the rotation of the sensor node itself. Either sufficient rotated examples have to be provided or an invariance transform has to be added in future improvements.

Clearly, a trade-off in result quality and resource demand is offered by the different proposed methods. This is illustrated in Figures 17 and 18 by putting the performance and required cost for both methods D2D and V2C side by side for RBF and SVR, respectively. This looks on first sight too much in favor of D2D, as existing overhead of computing 
the distances from magnetic measurements and computing coordinates from transformed distances is not yet included in the given effort calculation. V2C approach does not need these steps at all. The final choice of method depends on the applications requirements on localization accuracy, allowable resource expenses, and the potential need for node-based localization computation during measurement.

\section{Conclusion}

This paper presented our work on a artificial neural network based enhancement of a dedicated magnetic localization system for a wireless integrated autonomous sensor swarm for distributed process parameter measurement in industrial environment, such as, for example, large scale stainless containers or fermentation tanks. The concept has no limitation on the sensor swarm size. The focus of our work was on the improvement of the initially achieved mediocre localization accuracy of the basic electronic system by means of a learning system based on neural virtual sensors adapting to the regarded scenario and system instance. RBF and SVR techniques were investigated in D2D, along with standard and advanced distance to coordinate computation and V2C approaches for three different demonstrators from lab to industrial scale.

The supervised learning approach achieved significant improvements, even for uncalibrated sensors and measurement set-up in all investigated configurations. The most fortunate method combination of D2D by SVR followed by PSO-based distance to coordinate computation returned a factor large than 4 of improvement for the BREW data from industrial scenario. The mean error of about $3 \mathrm{~cm}$ is less than the size of the currently employed sensor node, bringing the localization results well in the order of the expectation met, for example, in brewery industry as well as in challenging smart-environment applications. However, the presented learning system could also be abstracted to the benefit of nonmagnetic, that is, RF-based, localization.

The mandatory synchronization for the pursued magnetic localization approach motivated the extension of the described approach by a learning SVM-based edge detector for coil switching time detection and sensor clock correction. Simulations on data from the HMI demonstrator showed the viability of the approach and, thus, the overall system.

Future work will improve the system with regard to artificial neural network size reduction, robustness issues, lean synchronization techniques, swarm visualization, self-x mechanisms, improved coil-switching power electronics, and $3 \mathrm{D}$ integration.

\section{Conflict of Interests}

The authors declare that there is no conflict of interests regarding the publication of this paper.

\section{Acknowledgments}

The authors would like to gratefully acknowledge the support of the German BMBF, mst-AVS, Project PAC4PT-ROSIG
Grant no. 16SV3604 for funding the baseline of this follow-up work and the contributions of L. Rao to the first-cut sensor node SW and of S. Carrella for collecting the raw data for the BREW data set in Warsteiner brewery together with D. Groben.

\section{References}

[1] T. Selker, Counter-intelligence project, 2008, http://www.media .mit.edu/ci/.

[2] B. Warneke, M. Scott, B. Leibowitz et al., "An autonomous 16 solar-powered node for distributed wireless sensor networks," in Proceedings of the 1st IEEE International Conference on Sensors (IEEE Sensors '02), vol. 2, pp. 1510-1515, June 2002.

[3] K. Pister, J. Kahn, and B. Boser, "Smart dust-autonomous sensing and communication in a cubic millimeter," 2001, http:// robotics.eecs.berkeley.edu/pister/SmartDust/.

[4] International Technology Roadmap for Semiconductors, http:// www.itrs.net/.

[5] W. Arden, M. Brillouët, P. Cogez, M. Graef, B. Huizing, and R. Mahnkopf, "more-than-moore," White paper, 2013, http:// www.itrs.net/Links/2010ITRS/IRC-ITRS-MtM-v2\%203.pdf.

[6] "Sensornetzwerke," Tech. Rep., 2013, http://www.izm.fraunhofer.de/.

[7] A. Reinecke, U. Pöpping, and U. Hampel, "Autonome Sensorpartikel zur räumlichen Parametererfassung in großskaligen Behältern," in GMA/ITG-Fachtagung Sensoren und Messsysteme, pp. 513-521, VDE, June 2012.

[8] C. V. Nelson and B. C. Jacobs, "Magnetic sensor system for fastresponse, high resolution, high accuracy three-dimensional position measurements," PCT patent application WO 2000017 603A1, applicant: The John Hopkins University, USA, 1998.

[9] J. S. Bladen and A. P. Anderson, "Position location system," PCT patent application WO 1994004 938A1, August 14, 1992, applicant for all states except US: British Telecommunications Pub. Ltd. US: Inventors.

[10] Motilis, Innovative Pill Technology, 2009, http://www.motilis .com/.

[11] Matesy GmbH, 3d-magtrack, 3d-magma, http://www.matesy de/de/.

[12] Polhemus,2012,http://www.polhemus.com/?page/MilitaryWhyMagneticTracking.

[13] Ascension Technology Corporation Products Application, October 2014, http://www.ascension-tech.com/medical/index .php.

[14] "Vector project," 2010, http://www.vector-project.com/.

[15] G. Pirkl and P. Lukowicz, "Robust, low cost indoor positioning using magnetic resonant coupling," in Proceedings of the ACM Conference on Ubiquitous Computing (UbiComp '12), pp. 431440, ACM Press, New York, NY, USA, 2012.

[16] M. Barry, A. Grnerbl, and P. Lukowicz, "Wearable jointangle measurement with modulated magnetic field from $1 / \mathrm{c}$ oscilators," in Proceedings of the 4th International Workshop on Wearable and Implantable Body Sensor Networks (BSN '07), S. Leonhardt, T. Falck, and P. Mhnen, Eds., vol. 13, chapter 7 of IFMBE Proceedings, pp. 43-48, Springer, New York, NY, USA, 2007.

[17] J. Blankenbach and A. Norrdine, "Position estimation using artificial generated magnetic fields," in Proceedings of the International Conference on Indoor Positioning and Indoor Navigation (IPIN '10), pp. 1-5, September 2010. 
[18] J. Blankenbach, A. Norrdine, and H. Hellmers, "Adaptive signal processing for a magnetic indoor positioning system," in Proceedings of the International Conference on Indoor Positioning and Indoor Navigation (IPIN '10), Short paper, pp. 15-17, 2010.

[19] J. Ágila, B. Link, G. Fabritius, M. H. Alizai, and K. Wehrle, "Burrow view-seeing the world through the eyes of rats," in Proceedings of the IEEE International Workshop on Information Quality and Quality of Service for Pervasive Computing IQ2S.

[20] Asensor Technologies AB, He444 Series 3d Analog Hall Sensors, Datasheet, SENSOR+TEST 2013, Asensor Technologies AB, Nürnberg, Germany, 2013.

[21] Bosch Sensortec, BMX055, BNO055, 2013, http://www.boschsensortec.com/en/homepage/products_3/9_axis_sensors_5/9axis_sensors.

[22] Invensense, "MPU-9150 Nine-Axis (Gyro + Accelerometer + Compass) MEMS Motion Tracking Device," 2013, http://www .invensense.com/mems/gyro/mpu9150.html.

[23] Sensitec, "AFF755B datasheet," 2011, http://www.sensitec.com/ english/products/magnetic-field/aff755.html.

[24] A. K, D. Groben, A. C. Kammara, and K. Thongpull, "3d localization of low-power wireless sensor nodes based on amr sensors in industrial and ami applications," in Proceedings of the 16th International Conference on Sensors and Measurement Technology (SENSORS '13), pp. 346-351, AMA ServiceGmbH, Nürnberg, Germany, May 2013.

[25] "Hofmann Leiterplatten GmbH," 2013, http://www.hofmann .de/.

[26] R. Akl, K. Pasupathy, and M. Haidar, "Anchor nodes placement for effective passive localization," in Proceedings of the International Conference on Selected Topics in Mobile and Wireless Networking (iCOST '11), pp. 127-132, October 2011.

[27] B. Tatham and T. Kunz, "Anchor node placement for localization in wireless sensor networks," in Proceeedings of the 7th IEEE International Conference on Wireless and Mobile Computing, Networking and Communications (WiMob '11), pp. 180-187, October 2011.

[28] S. Carrella, K. Iswandy, and A. König, "A system for localization of wireless sensor nodes in industrial applications based on sequentially emitted magnetic fields sensed by tri-axial amr sensors," in Proceedings of the 11th Symposium Magneto-Resistive Sensors and Magnetic Systems, Lahnau, Germany, March 2011.

[29] M. Leonardi, A. Mathias, and G. Galati, "Two efficient localization algorithms for multilateration," International Journal of Microwave and Wireless Technologies, vol. 1, no. 3, pp. 223-229, 2009.

[30] A. Wessels, X. Wang, R. Laur, and W. Lang, "Dynamic indoor localization using multilateration with RSSI in wireless sensor networks for transport logistics," Procedia Engineering, vol. 5, pp. 220-223, 2010.

[31] Many Authors, "Multilateration and ADS-B an executive reference guide," 2013, http://www.multilateration.com/resources .html.

[32] K. Iswandy and A. König, "Soft-computing techniques to advance nonlinear mappings for multi-variate data visualization and wireless sensor localization," e-Newsletter IEEE SMC Society, vol. 29, 2009.

[33] A. König, "Interactive visualization and analysis of hierarchical neural projections for data mining," IEEE Transactions on Neural Networks, vol. 11, no. 3, pp. 615-624, 2000.

[34] A. C. Kammara and A. König, "Advanced methods for 3D magnetic localization in industrial process distributed datalogging with a sparse distance matrix," in Soft Computing in Industrial Applications, vol. 223 of Advances in Intelligent Systems and Computing, pp. 149-156, Springer, Berlin, Germany, 2014.

[35] J. Kennedy and R. Eberhart, "Particle swarm optimization," in Proceedings of the IEEE International Conference on Neural Networks, vol. 4, pp. 1942-1948, December 1995.

[36] J. Cioffi, W. Abbott, H. Thapar, C. Melas, and K. D. Fisher, "Adaptive equalization in magnetic-disk storage channels," IEEE Communications Magazine, vol. 28, no. 2, pp. 14-29, 1990.

[37] K. Iswandy and A. König, "Hybrid virtual sensor based on RBFN or SVR compared for an embedded application," in Knowlege-Based and Intelligent Information and Engineering Systems, A. König, A. Dengel, K. Hinkelmann, K. Kise, R. Howlett, and L. Jain, Eds., vol. 6882 of Lecture Notes in Computer Science, chapter 34, pp. 335-344, Springer, 2011.

[38] H. Pasika, S. Haykin, E. Clothiaux, and R. Stewart, "Neural networks for sensor fusion in remote sensing," in Proceedings of the International Joint Conference on Neural Networks (IJCNN '99), vol. 4, pp. 2772-2776, 1999.

[39] S. Haykin, Neural Networks, edited by S. Haykin, Macmillan, New York, NY, USA, 1994.

[40] P. D. Wasserman, Advanced Methods in Neural Computing, Van Nostrand Reinhold, New York, NY, USA, 1st edition, 1993.

[41] J. Platt, "A resource-allocating network for function interpolation," Neural Computation, vol. 3, no. 2, pp. 213-225, 1991.

[42] A. J. Smola and B. Schölkopf, "A tutorial on support vector regression," Statistics and Computing, vol. 14, no. 3, pp. 199-222, 2004.

[43] C.-C. Chang and C.-J. Lin, A practical guide to support vector classification, 2010, http://www.csie.ntu.edu.tw/ cjlin/papers/ guide/guide.pdf.

[44] C.-C. Chang and C.-J. Lin, "Libsvm: a library for support vector machines," ACM Transactions on Intelligent Systems and Technology, vol. 2, no. 3, pp. 27:1-27:27, 2011. 

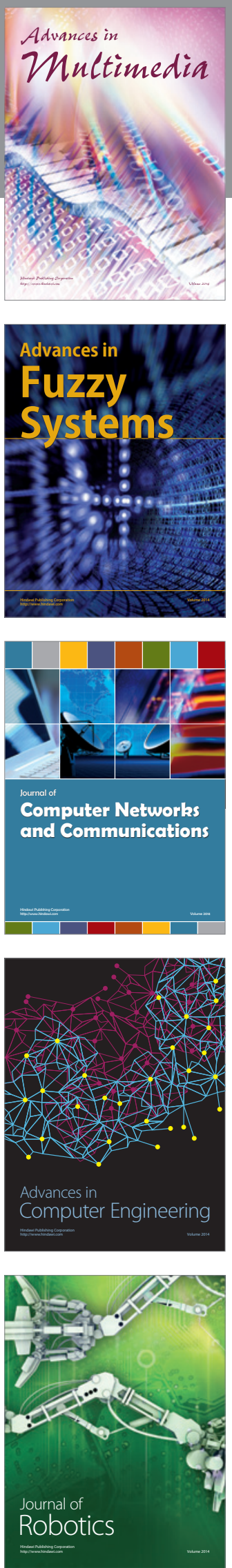

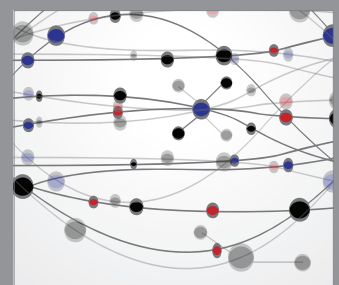

The Scientific World Journal
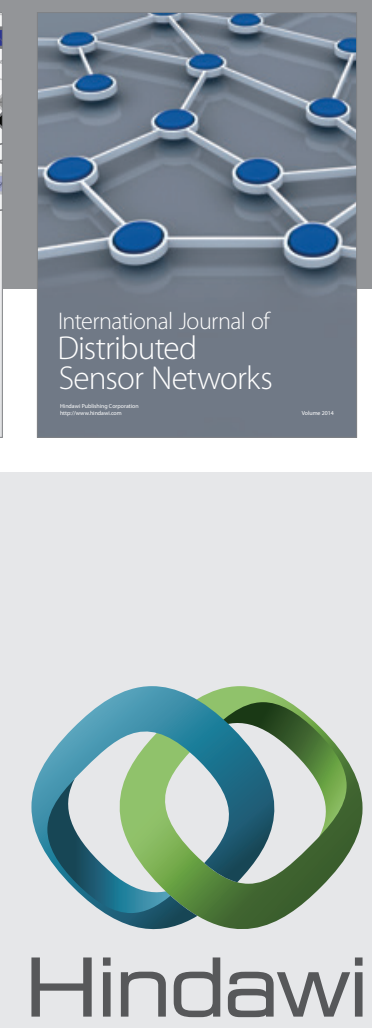

Submit your manuscripts at

http://www.hindawi.com
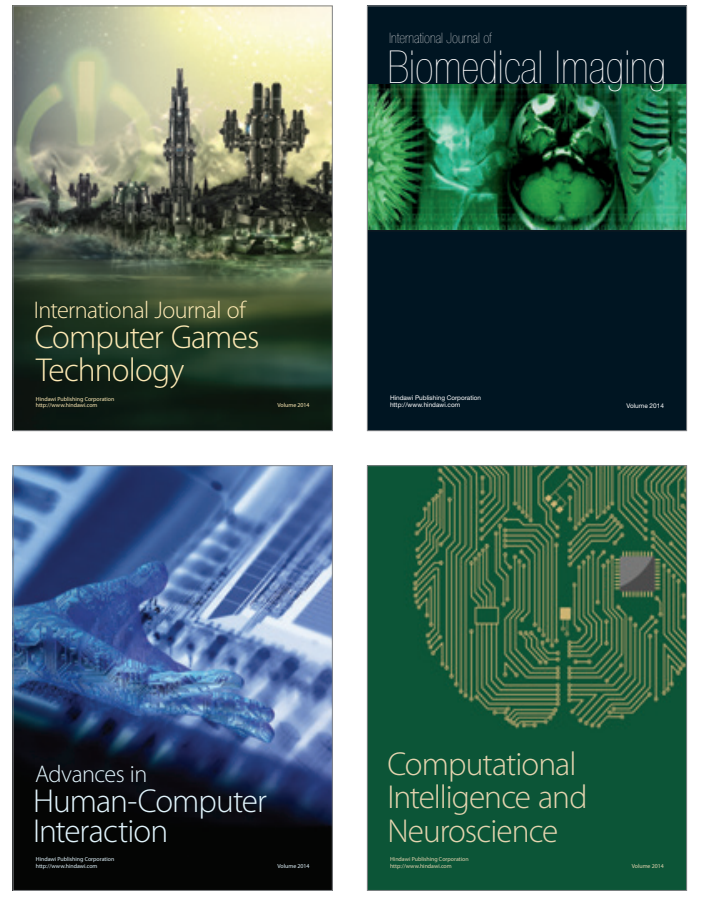
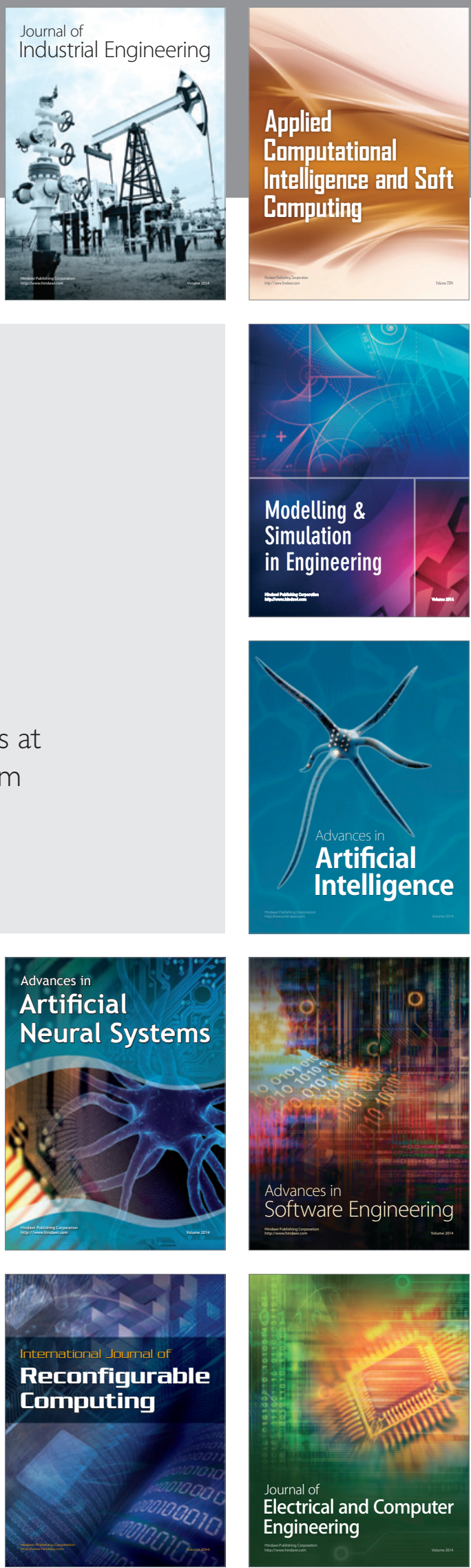